\title{
Hypertension in African Populations: Review and Computational Insights
}

\author{
Sihle E. Mabhida ${ }^{1,2,+}$, Lebohang Mashatola ${ }^{3,+}{ }^{+}$Mandeep Kaur ${ }^{3}{ }^{-}$, Jyoti R. Sharma ${ }^{1}$, Teke Apalata ${ }^{4}$, \\ Babu Muhamed ${ }^{5,6} \oplus^{(}$, Mongi Benjeddou ${ }^{2}$ and Rabia Johnson $1,7, * \mathbb{C}$
}

1 Biomedical Research and Innovation Platform, South African Medical Research Council, Tygerberg 7505, South Africa; sihlemabhida@gmail.com (S.E.M.); jyoti.sharma@mrc.ac.za (J.R.S.)

2 Department of Biotechnology, Faculty of Natural Science, University of the Western Cape, Private Bag X17, Bellville, Cape Town 7535, South Africa; mbenjeddo@uwc.ac.za

3 School of Molecular and Cell Biology, University of the Witwatersrand, Private Bag 3, Johannesburg 2050, South Africa; 681452@students.wits.ac.za (L.M.); mandeep.kaur@wits.ac.za (M.K.)

4 Division of Medical Microbiology, Department of Laboratory-Medicine and Pathology, Faculty of Health Sciences, Walter Sisulu University and National Health Laboratory Services, Mthatha 5100, South Africa; tapalata@wsu.ac.za

5 Hatter Institute for Cardiovascular Diseases Research in Africa, Department of Medicine, University of Cape Town, Cape Town 7535, South Africa; babu.muhamed@uct.ac.za

6 Children's National Health System, Division of Cardiology, Washington, DC 20010, USA

7 Division of Medical Physiology, Faculty of Medicine and Health Sciences, Stellenbosch University, Tygerberg 7505, South Africa

* Correspondence: rabia.johnson@mrc.ac.za; Tel.: +27-21-938-0866

+ These authors contributed equally to this work.

Citation: Mabhida, S.E.; Mashatola, L.; Kaur, M.; Sharma, J.R.; Apalata, T.; Muhamed, B.; Benjeddou, M.; Johnson, R. Hypertension in African Populations: Review and Computational Insights. Genes 2021, 12, 532. https://doi.org/10.3390/ genes12040532

Academic Editor: Donato Gemmati

Received: 19 February 2021

Accepted: 18 March 2021

Published: 6 April 2021

Publisher's Note: MDPI stays neutral with regard to jurisdictional claims in published maps and institutional affiliations.

Copyright: (c) 2021 by the authors. Licensee MDPI, Basel, Switzerland. This article is an open access article distributed under the terms and conditions of the Creative Commons Attribution (CC BY) license (https:// creativecommons.org/licenses/by/ $4.0 /)$.
Abstract: Hypertension (HTN) is a persistent public health problem affecting approximately 1.3 billion individuals globally. Treatment-resistant hypertension (TRH) is defined as high blood pressure (BP) in a hypertensive patient that remains above goal despite use of $\geq 3$ antihypertensive agents of different classes including a diuretic. Despite a plethora of treatment options available, only $31.0 \%$ of individuals have their HTN controlled. Interindividual genetic variability to drug response might explain this disappointing outcome because of genetic polymorphisms. Additionally, the poor knowledge of pathophysiological mechanisms underlying hypertensive disease and the long-term interaction of antihypertensive drugs with blood pressure control mechanisms further aggravates the problem. Furthermore, in Africa, there is a paucity of pharmacogenomic data on the treatment of resistant hypertension. Therefore, identification of genetic signals having the potential to predict the response of a drug for a given individual in an African population has been the subject of intensive investigation. In this review, we aim to systematically extract and discuss African evidence on the genetic variation, and pharmacogenomics towards the treatment of HTN. Furthermore, in silico methods are utilized to elucidate biological processes that will aid in identifying novel drug targets for the treatment of resistant hypertension in an African population. To provide an expanded view of genetic variants associated with the development of HTN, this study was performed using publicly available databases such as PubMed, Scopus, Web of Science, African Journal Online, PharmGKB searching for relevant papers between 1984 and 2020. A total of 2784 articles were reviewed, and only 42 studies were included following the inclusion criteria. Twenty studies reported associations with HTN and genes such as AGT (rs699), ACE (rs1799752), NOS3 (rs1799983), MTHFR (rs1801133), AGTR1 (rs5186), while twenty-two studies did not show any association within the African population. Thereafter, an in silico predictive approach was utilized to identify several genes including CLCNKB, CYPB11B2, SH2B2, STK9, and TBX5 which may act as potential drug targets because they are involved in pathways known to influence blood pressure. Next, co-expressed genes were identified as they are controlled by the same transcriptional regulatory program and may potentially be more effective as multiple drug targets in the treatment regimens for HTN. Genes belonging to the co-expressed gene cluster, ACE, AGT, AGTR1, AGTR2, and NOS3 as well as CSK and ADRG1 showed enrichment of G-protein-coupled receptor activity, the classical targets of drug discovery, which mediate cellular signaling processes. The latter is of importance, as the targeting of co-regulatory 
gene clusters will allow for the development of more effective HTN drug targets that could decrease the prevalence of both controlled and TRH.

Keywords: hypertension; pharmacogenomics; single-nucleotide polymorphism; Africa; genetic variation

\section{Introduction}

Hypertension (HTN), also known as high blood pressure (BP), can be classified into primary or secondary HTN with secondary HTN affecting 5-10\% of hypertensive patients with the cause linked to an underlying medical condition. In contrast, primary hypertension also known as essential HTN accounts for $90-95 \%$ of HTN cases, it has no identifiable secondary root and is the most common cause of stroke and cardiovascular disease (CVD) [1,2]. Accumulative evidence showed that the prevalence of HTN is 1.3 billion, and this number is projected to increase to 1.56 billion by 2030 with an estimated global economic cost of $\$ 274$ billion [1-4]. Despite the availability of advanced diagnostic options and the use of multiple antihypertensive medications, many studies have reported inadequate control of blood pressure among hypertensive individuals worldwide, including Africa [2,5-7]. This inability to control blood pressure even after adherence has been referred to as treatment-resistant hypertension (TRH). According to the American heart association, TRH is defined as a BP which remains $\geq 140 / 90 \mathrm{mg}$ despite the use of three or more antihypertensive drugs [8,9].

Although poor control of high blood pressure may have many causes, the most likely contributor is the poor response to the prescribed antihypertensive drug [10]. Current drugs are used to treat HTN without an in-depth understanding of the biological basis that regulates the disease or the effect of an individual's make-up on the efficacy of the drug to control HTN [11]. The most commonly prescribed drug classes to treat hypertension include diuretics and vasodilators designed to reduce vascular resistance. The major disadvantages are side effects (i.e., renal dysfunction) and unpredicted blood pressure responses in patients (i.e., HTN) [12,13]. Drug classes developed from the knowledge of biological pathways include the angiotensin II antagonists and angiotensin-converting enzyme inhibitors. These have been developed through the understanding of the reninangiotensin-aldosterone system (RAAS) in the regulation of blood pressure. As such, first-line therapy includes angiotensin-converting enzyme $(A C E)$ inhibitors, angiotensin II receptors, and calcium-channel blockers [14,15]. These have proven to be the most effective drug classes (coupled with lower side effects) when used as a combination therapy [16]. Nonetheless, though effective, the one-drug-fits-all model has been proven to be ineffective due to an individual's genetic make-up and variable response to several antihypertensive drugs could be attributed to the genetic variability in the candidate BP regulating genes and their pathways [17]. Many studies have demonstrated that genetic factors are responsible not only for blood pressure elevation but also play a profound role in the interindividual variability in drug response, offering an opportunity for pharmacogenomic investigation and potential individualized drug therapy $[18,19]$. Therefore, it has been speculated that a patient's inter-individual genetic variation can be used to understand the pathophysiology of the disease and that this pharmacogenomics approach may have the potential of individualizing HTN drug therapy based on the patient's genetic background [20,21]. For example, Guo et al., [22] and Choi et al., [23] have reported successful examples of genetic polymorphisms on blood pressure response to antihypertensive therapies. Recently, Ma et al. [21] reported that various informative genetic variants can be utilized for the identification of subtypes of hypertension. These studies have highlighted the importance of understanding the biological basis of the disease and the contribution of genetic variations in the development and progression of the disease. 
Despite major advances being made over the last decade, understanding the role of genetics governing the phenotypic state of HTN is complex because on one hand there are rare monogenic hypertensive syndromes while on the other side are the cases of primary or TRH, which may occur due to a varied expression and interactions of multiple genes [24]. However, polygenic inheritance patterns in such cases are not only complex but also enigmatic.

Nonetheless, various subtle genetic variations have been identified through approaches such as genome-wide association studies, and these small variations among individuals may account for genetic susceptibility to HTN. However, these single-nucleotide polymorphisms (SNP) have been shown to vary across racial and ethnic groups and play a pivotal role during the development and progression of this phenotypic state [25]. SNPs are changes in specific nucleotides at fixed positions in DNA sequence and occur frequently once in every 1000 base pairs. These are the most commonly occurring genetic variations causing varied inter-individual drug response which remains a major public health concern $[1,3,26]$. Furthermore, it has been speculated that variable response to drugs could also be due to different subtypes of a disease phenotype in certain individuals. To exemplify, patients demonstrating better BP response with certain drugs may have different pathophysiology of HTN than others showing poor response with those drugs [18]. Therefore, a thorough understanding of the genetic background of HTN is critical to predict an individual's disease risk as well as to improve individualized treatment response. Despite many SNPs having no clinical significance, several key SNPs have been reported with a detailed analysis through pharmacogenomics-based studies playing an important role in antihypertensive drug response [25,27].

As such, the purpose of this review was to systematically extract and discuss African evidence on the effect of genetic variations in hypertensive patients and use in silico methods to further elucidate biological processes that will aid in identifying novel drug targets for the treatment of HTN in an African population.

\section{Materials and Methods}

A systematic search was performed using subject headings or primary search terms such as resistant hypertension, genetics, SNP, and pharmacogenomic under the Preferred Reporting Items for Systematic Review (PRISMA) guidelines [28]. This was done using major search engines and databases such as PubMed, Scopus, Web of Science, African Journal Online, and PharmGKB were used for the search as shown in Table S1.

\subsection{Inclusion Criteria and Data Extraction}

The studies were included in the systemic review based on the following criteria: (i) Investigated the association between SNPs and HTN in African-based population, (ii) Used case-control design, (iii) Published from 1984 to 2020, (iv) Studies done on humans (Table 1). Studies were excluded if duplicate publications reported selectively on migrant Africans outside Africa, family studies, used linkage analysis and analyzed mixed populations of African descent without considering their country of residence. Moreover, language restriction was applied with studies conducted in languages other than English were excluded. The articles were independently assessed for compliance with the inclusion or exclusion criteria by two authors resolving disagreements and reached a consistent decision. The following information was extracted from each study: first author and year of publication; country of origin; ethnicity of the study population; the number of subjects under hypertensive cases and controls; diagnostic criteria for hypertensive cases and controls and SNPs analyzed. 
Table 1. Inclusion Criteria and Data Extraction.

\begin{tabular}{cc}
\hline Inclusion & Exclusion \\
\hline African population & Studies in non-African countries \\
\hline Published from 1984 to 2020 & Studies conducted before 1983 \\
\hline Human studies & Non-human studies \\
\hline $\begin{array}{c}\text { Studies investigating an association }(p<0.05) \text { between } \\
\text { SNP and hypertension. }\end{array}$ & Studies in gene expression \\
\hline Case-control design & Reviews \\
\hline
\end{tabular}

\subsection{Drug-Gene Interaction}

The bioinformatics tool iCTNET from Cytoscape (available at http:/ / apps.cytoscape. org/apps/iCTNET (accessed on 10 March 2020) was used to search through the DrugBank (available at https:/ / www.drugbank.ca (accessed on 10 March 2020) and comparative toxicogenomic database (CTD) (available at http:/ / ctdbase.org/ (accessed on 10 March 2020) to identify drug-gene interactions. These were coupled with the occurring frequent and infrequent side-effects for each drug and plotted in a network. This analysis helped to identify the common drug-targets of HTN.

\subsection{Co-Expression Networks}

Networks of co-expressed genes were generated from mathematical models which were used to predict protein-protein interactions (PPIs) using the bioinformatics tool Expression Correlation from Cytoscape [29] (available at http:/ /apps.cytoscape.org/apps/ Expression Correlation (accessed on 9 March 2020). The reference database Expression Correlation utilizes sample data from the human gene atlas project (available at https:/ / www.pnas.org/content/101/16/6062 (accessed on 9 March 2020) from 158 expression experiments on healthy humans. From the input gene list, Expression Correlation computes a similarity matrix using expression levels from the reference database. Genegene correlation networks are then computed and visualized as an interaction network.

The biological function was related to the identified co-expressed gene clusters using the gene ontology (GO) database for molecular function. This allowed for the selection of specific gene-clusters that might pose as drug targets.

\subsection{Biological Functional Enrichment}

To perform biological functional enrichment, the R Bioconductor tool Cluster Profiler [30] was used to identify enriched GO, Kyoto Encyclopedia of Genes and Genomes (KEGG) pathways, and disease ontologies. Fisher's exact test was performed to identify significantly enriched terms with a Benjamin-Hochberg adjusted probability value (BH adjusted $p$-value) of less than 0.05 . This was conducted to relate biological function to the gene list related to HTN. Selecting genes as potential drug-targets with minimal influence on other biological pathways and processes was imperative to minimizing side effects in HTN patients.

\subsection{Disease Ontology Enrichment}

To identify and link the disease state to known HTN genes, disease ontology enrichment (DO) was performed using Cluster Profiler [30]. Disease ontology enrichment involved cross-referencing mappings to the Medical Subject Headings (MeSH) (available at https: / / www.nlm.nih.gov / databases (accessed on 9 March 2020), International Classification of Disease (ICD) (available at https://www.cdc.gov/nchs/icd/ (accessed on 9 March 2020), National Cancer Institute (NCI), (available at https:/ /www.cancer.gov / research/resources (accessed on 9 March 2020), Systematized Nomenclature of MedicineClinical Terms (SNOMED CT) (available at http:/ / www.snomed.org (accessed on 9 March 
2020) and Online Mendelian Inheritance in Man (OMIM) (available at https:/ /www.omim. org (accessed on 9 March 2020) databases. A hypergeometric model assessed the overrepresentation of the selected genes and their association with a disease. A BH-adjusted $p$-value was calculated to identify significantly associated diseases ( $\mathrm{BH} p$-value less than 0.05 ). This was performed to further identify possible HTN genes that show no association with other diseases. This was a possible indicator that the targeting chosen genes would have minimal interference with other biological functions.

\section{Results}

\subsection{Selected Studies}

We identified 2784 studies through data searches: PubMed $(n=410)$, Web of Science $(n=1115)$, Scopus $(n=1245)$, PharmGKB $(n=11)$, and African Journal Online $(n=3)$ (Figure 1). After removing duplicates $(n=1222)$ and studies that were conducted before molecular biology techniques $(n=43)$ the full text of 1519 publications were tested for suitability [28]. Of these, 1347 were excluded as follows; non-human studies $(n=29)$, non-HTN studies $(n=1194)$, non-African countries $(n=78)$, reviews $(n=46)$, and different study design (e.g., investigated the expression of genes in patients with HTN, clinical studies on HTN and family-based studies) $(n=130)$. Finally, 42 studies were included in this review.

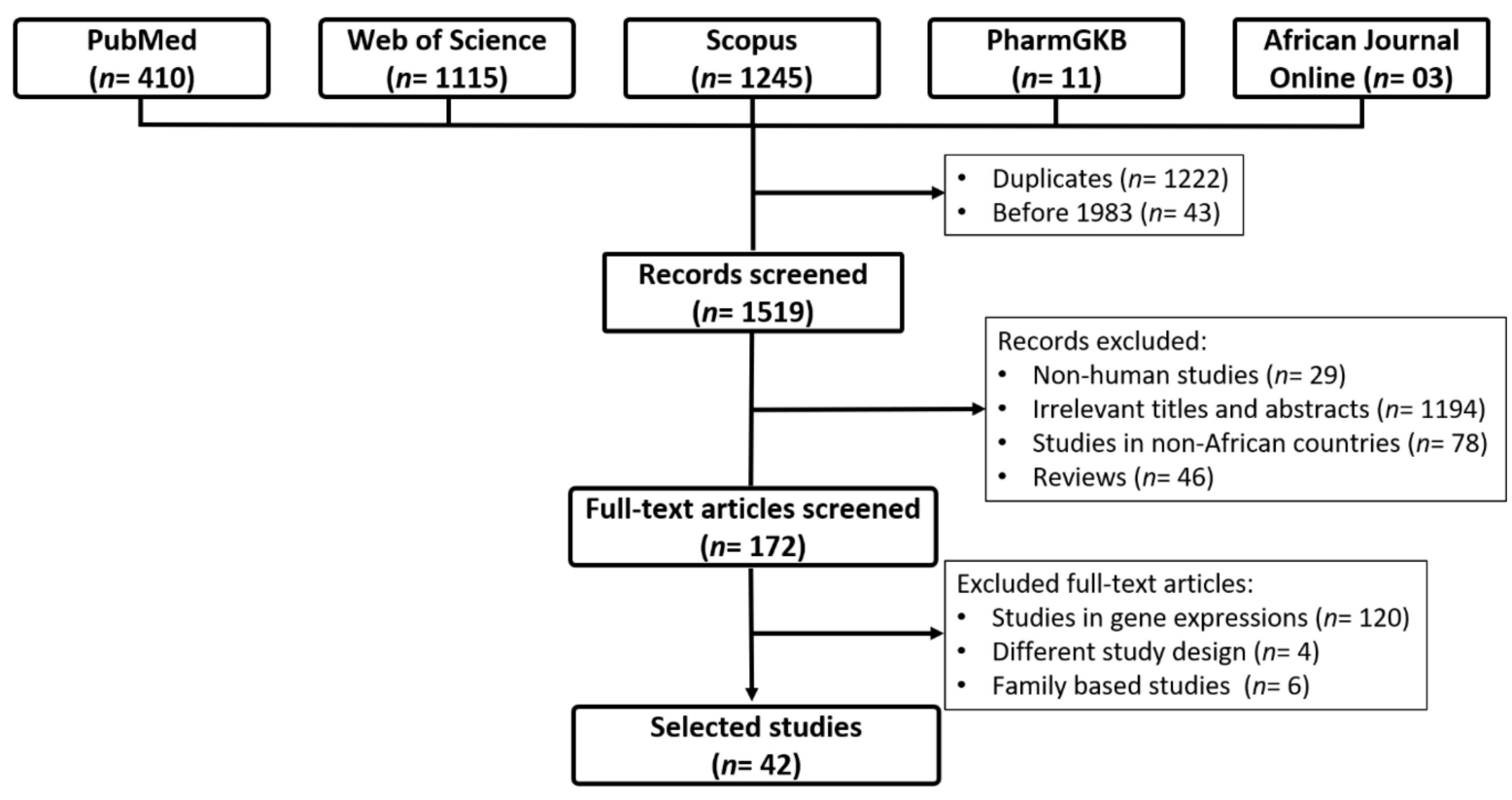

Figure 1. Flowchart for the study selection.

The baseline characteristics of the included studies are summarized in Table 2. HTN was defined as systolic/diastolic BP (SBP/DBP) $\geq 140 / 90 \mathrm{~mm} \mathrm{Hg}$ or the use of antihypertensive medications at inclusion [31,32]. Other studies used auscultatory DBP $>90 \mathrm{~mm}$ $\mathrm{Hg}$ or 24-h ambulatory DBP $>85 \mathrm{~mm} \mathrm{Hg}$ and auscultatory DBP $>95 \mathrm{~mm} \mathrm{Hg}$, SBP/DBP > 159/80 $\mathrm{mm} \mathrm{Hg}$, SBP/DBP >139/89 mm Hg and SBP/DBP $\geq 125 / 80 \mathrm{~mm} \mathrm{Hg}$, 25. This review did not identify a genome-wide association study (GWAS) conducted among the African population with HTN. 
Table 2. Hypertension and single-nucleotide polymorphisms (SNPs) association in Africa.

\begin{tabular}{|c|c|c|c|c|c|c|c|c|c|c|}
\hline Gene & $\begin{array}{c}\text { Chr } \\
\text { Position }\end{array}$ & SNP & Alleles & $\begin{array}{c}\text { Alt Allele Freq, } \\
\text { Global (db. SNP) }\end{array}$ & $\begin{array}{c}\text { Alt Allele Freq, } \\
\text { African (db. SNP) }\end{array}$ & Cases No. & Controls & $\begin{array}{l}\text { Association } \\
(p<0.05)\end{array}$ & Country & Author Year \\
\hline \multirow[t]{8}{*}{$A G T$} & $1 q 42.2$ & rs2004776 & $C>G$ & 0.410 & 0.487 & 782 & 2099 & Yes & Uganda & Kayima et al., 2017 [33] \\
\hline & & rs4762 & $\mathrm{G}>\mathrm{A}$ & 0.102 & 0.054 & 75 & 70 & No & Algeria & Amrani et al., 2015 [34] \\
\hline & & rs699 & $A>G$ & 0.705 & 0.903 & 202 & 204 & No & Burkina Faso & Tchelougou et al., 2015 [35] \\
\hline & & & & & & 612 & 612 & No & Nigeria & Kooffreh et al., 2014 [36] \\
\hline & & & & & & 81 & 178 & No & Algeria & Meroufel et al., 2014 [37] \\
\hline & & & & & & 110 & 93 & No & Egypt & AbdRaboh et al. 2012 [38] \\
\hline & & & & & & 39 & 22 & No & Tunisia & ALrefai et al., 2010 [39] \\
\hline & & & & & & 195 & 107 & No & South African & Ranjith et al., 2004 [40] \\
\hline \multirow[t]{7}{*}{ AGTR1 } & $3 q 24$ & rs5186 & $\mathrm{A}>\mathrm{C}$ & 0.118 & 0.020 & 36 & 50 & No & Cameroon & Ghogomu et al., 2016 [41] \\
\hline & & & & & & 202 & 204 & No & Burkina Faso & Tchelougou et al., 2015 [35] \\
\hline & & & & & & 81 & 178 & No & Algeria & Meroufel et al., 2014 [37] \\
\hline & & & & & & 612 & 612 & No & Nigeria & Kooffreh et al., 2014 [36] \\
\hline & & & & & & 142 & 191 & No & Tunisia & Mehri et al., 2012 [38] \\
\hline & & & & & & 195 & 107 & No & South African & Ranjith et al., 2004 [40] \\
\hline & & & & & & $\mathrm{NA}$ & NA & Yes & Ghana & Williams et al., 2004 [43] \\
\hline \multirow[t]{7}{*}{$A C E$} & $17 q 23.3$ & rs1799752 & NA & NA & NA & 202 & 204 & Yes & Burkina Faso & Tchelougou et al., 2015 [35] \\
\hline & & & & & & 217 & 161 & Yes & Egypt & Zawilla et al., 2014 [44] \\
\hline & & & & & & 110 & 93 & No & Egypt & AbdRaboh et al. 2012 \\
\hline & & & & & & 40 & 21 & Yes & Egypt & Badr et al., 2012 [45] \\
\hline & & & & & & 142 & 191 & No & Tunisia & Mehri et al., 2012 [38] \\
\hline & & & & & & 40 & 40 & Yes & Egypt & Bessa et al., 2009 [46] \\
\hline & & & & & & 195 & 107 & No & South African & Ranjith et al., 2004 [40] \\
\hline \multirow[t]{2}{*}{ NOS3 } & $7 \mathrm{q} 36.1$ & rs1799983 & $\mathrm{T}>\mathrm{A}$ & 0.824 & 0.930 & 77 & 77 & Yes & Algeria & Amrani-Midoun et al., 2019 [47] \\
\hline & & & & & & 145 & 184 & Yes & Morocco & Nassereddine et al., 2018 [25] \\
\hline
\end{tabular}


Table 2. Cont

\begin{tabular}{|c|c|c|c|c|c|c|c|c|c|c|}
\hline Gene & $\begin{array}{c}\text { Chr } \\
\text { Position }\end{array}$ & SNP & Alleles & $\begin{array}{c}\text { Alt Allele Freq, } \\
\text { Global (db. SNP) }\end{array}$ & $\begin{array}{c}\text { Alt Allele Freq, } \\
\text { African (db. SNP) }\end{array}$ & Cases No. & Controls & $\begin{array}{l}\text { Association } \\
(p<0.05)\end{array}$ & Country & Author Year \\
\hline & & $\begin{array}{c}\text { rs2070744 } \\
\text { intron } 4 \\
\text { VNTR }\end{array}$ & $\begin{array}{l}\mathrm{C}>\mathrm{T} \\
\mathrm{N} / \mathrm{A}\end{array}$ & $\begin{array}{l}0.766 \\
\text { N/A }\end{array}$ & $\begin{array}{l}0.862 \\
\text { N/A }\end{array}$ & 157 & 144 & $\begin{array}{l}\text { No } \\
\text { No } \\
\text { Yes }\end{array}$ & Sudan & Gamil et al., 2017 [48] \\
\hline & & rs1799983 & $\mathrm{T}>\mathrm{A}$ & 0.824 & 0.930 & 70 & 30 & Yes & Tunisia & ALrefai et al., 2016 [39] \\
\hline & & rs2070744 & $\mathrm{C}>\mathrm{T}$ & 0.766 & 0.862 & 288 & 373 & Yes & Tunisia & Jemaa et al., 2011 [49] \\
\hline & & rs1799983 & NA & NA & NA & 537 & 565 & No & Tunisia & Sediri et al., 2010 [50] \\
\hline \multirow[t]{4}{*}{ MTHFR } & $1 \mathrm{p} 36.3$ & rs1801133 & $\mathrm{G}>\mathrm{A}$ & 0.245 & 0.090 & 82 & 72 & No & Algeria & Amrani-Midoun et al., 2016 [52] \\
\hline & & & & & & 189 & 598 & Yes & Algeria & Lardjam-Hetraf et al.,2015 [53] \\
\hline & & & & & & 101 & 102 & Yes & Morocco & Nassereddine et al., 2015 [54] \\
\hline & & & & & & 97 & 84 & No & Egypt & Amin et al., 2012 [55] \\
\hline \multirow[t]{2}{*}{ AТР2B1 } & $12 q 21 . q 23$ & $\begin{array}{l}\text { rs2681472 } \\
\text { rs17249754 }\end{array}$ & $\begin{array}{l}A>G \\
G>A\end{array}$ & $\begin{array}{l}0.199 \\
0.209\end{array}$ & $\begin{array}{l}0.094 \\
0.131\end{array}$ & 180 & 200 & $\begin{array}{l}\text { Yes } \\
\text { Yes }\end{array}$ & Burkina Faso & Sombie et al., 2019 [3] \\
\hline & & rs2681492 & $\mathrm{T}>\mathrm{C}$ & 0.208 & 0.126 & 782 & 2099 & Yes & Uganda & Kayima et al., 2017 [33] \\
\hline \multirow[t]{2}{*}{ CLCNKB } & $1 \mathrm{p} 36.3$ & rs12140311 & $\mathrm{A}>\mathrm{T}$ & 0.098 & 0.214 & 213 & 545 & Yes & Ghana & Sile et al., 2009 [56] \\
\hline & & rs34561376 & $G>A$ & 0.082 & 0.142 & 213 & 545 & No & Ghana & Sile et al., 2007 [57] \\
\hline \multirow[t]{2}{*}{ GNB3 } & 12p13.31 & rs5443 & NA & NA & NA & 388 & 425 & No & Tunisia & Kabadou et al., 2013 [31] \\
\hline & & rs74837985 & $\mathrm{NA}$ & $\mathrm{NA}$ & $\mathrm{NA}$ & 40 & 40 & Yes & Egypt & Bessa et al., 2009 [46] \\
\hline \multirow[t]{2}{*}{ CNNM2 } & $10 \mathrm{q} 24.32$ & rs11191548 & $\mathrm{T}>\mathrm{C}$ & 0.152 & 0.025 & 782 & 2099 & Yes & Uganda & Kayima et al., 2017 [33] \\
\hline & & & & & & 189 & 598 & Yes & Algeria & Lardjam-Hetraf et al.,2015 [53] \\
\hline \multirow[t]{2}{*}{ PLEKHA7 } & 11p15.2 & rs381815 & $\mathrm{C}>\mathrm{A}$ & 0.206 & 0.190 & 782 & 2099 & Yes & Uganda & Kayima et al., 2017 [33] \\
\hline & & & & & & 189 & 598 & Yes & Algeria & Lardjam-Hetraf et al.,2015 [53] \\
\hline
\end{tabular}


Table 2. Cont.

\begin{tabular}{|c|c|c|c|c|c|c|c|c|c|c|}
\hline Gene & $\begin{array}{c}\text { Chr } \\
\text { Position }\end{array}$ & SNP & Alleles & $\begin{array}{c}\text { Alt Allele Freq, } \\
\text { Global (db. SNP) }\end{array}$ & $\begin{array}{l}\text { Alt Allele Freq, } \\
\text { African (db. SNP) }\end{array}$ & Cases No. & Controls & $\begin{array}{l}\text { Association } \\
(p<0.05)\end{array}$ & Country & Author Year \\
\hline \multirow[t]{2}{*}{$J A G 1$} & 20p12.2 & rs1327235 & $A>G$ & 0.464 & 0.494 & 782 & 2099 & Yes & Uganda & Kayima et al., 2017 [33] \\
\hline & & & & & & 189 & 598 & Yes & Algeria & Lardjam-Hetraf et al.,2015 [53] \\
\hline \multirow[t]{2}{*}{ SCNN1B } & $16 \mathrm{p} 12.2$ & rs149868979 & NA & NA & NA & 1468 & 471 & Yes & South Africa & Jones et al., 2012 [58] \\
\hline & & rs1799979 & $\mathrm{C}>\mathrm{T}$ & 0.007 & 0.024 & 519 & 514 & No & South Africa & Nkeh et al., 2003 [59] \\
\hline \multirow[t]{2}{*}{ FGF5 } & $4 \mathrm{q} 21.21$ & rs1458038 & $\mathrm{C}>\mathrm{T}$ & 0.230 & 0.037 & 782 & 2099 & Yes & Uganda & Kayima et al., 2017 [33] \\
\hline & & & & & & 189 & 598 & Yes & Algeria & Lardjam-Hetraf et al.,2015 [53] \\
\hline \multirow[t]{2}{*}{ EBF1 } & $5 q 33.3$ & rs11953630 & $\mathrm{C}>\mathrm{A}$ & 0.07 & 0.180 & 782 & 2099 & Yes & Uganda & Kayima et al., 2017 [33] \\
\hline & & & & & & 189 & 598 & Yes & Algeria & Lardjam-Hetraf et al.,2015 [53] \\
\hline STK39 & $2 \mathrm{q} 24.3$ & rs3754777 & $\mathrm{C}>\mathrm{T}$ & 0.195 & 0.119 & 180 & 200 & Yes & Burkina Faso & Sombie et al., 2019 [3] \\
\hline APOA5 & $11 \mathrm{q} 23.3$ & $\begin{array}{c}\text { rs662799 } \\
\text { rs3135506 } \\
\text { rs2075291 }\end{array}$ & $\begin{array}{l}\mathrm{G}>\mathrm{A} \\
\mathrm{G}>\mathrm{A} \\
\mathrm{C}>\mathrm{A}\end{array}$ & $\begin{array}{l}0.837 \\
0.056 \\
0.011\end{array}$ & $\begin{array}{l}0.884 \\
0.067 \\
0.002\end{array}$ & 149 & 134 & Yes & Morocco & Ouatou et al., 2014 [60] \\
\hline $\begin{array}{l}\text { CDKAL1 } \\
\text { IGF2BP2 }\end{array}$ & $\begin{array}{l}6 \mathrm{p} 22.3 \\
3 \mathrm{q} 27.2\end{array}$ & $\begin{array}{l}\text { rs7756992 } \\
\text { rs4402960 }\end{array}$ & $\begin{array}{l}\mathrm{A}>\mathrm{G} \\
\mathrm{G}>\mathrm{T}\end{array}$ & $\begin{array}{l}0.413 \\
0.389\end{array}$ & $\begin{array}{l}0.633 \\
0.567\end{array}$ & 200 & 208 & No & Tunisia & Lasram et al., 2015 [61] \\
\hline$B 2$ & $\begin{array}{l}\text { 14q32.1- } \\
\text { q32.2 }\end{array}$ & $\begin{array}{l}\mathrm{B}_{2} \mathrm{C}-58 \mathrm{~T} \\
\mathrm{~B}_{2}-9 /+9\end{array}$ & $\begin{array}{l}\text { NA } \\
\text { NA }\end{array}$ & $\begin{array}{l}\text { NA } \\
\text { NA }\end{array}$ & $\begin{array}{l}\text { NA } \\
\text { NA }\end{array}$ & 88 & 77 & Yes & South Africa & Moholisa et al., 2013 [63] \\
\hline CYP11B2 & $8 \mathrm{q} 24.3$ & rs1799998 & $A>G$ & 0.347 & 0.189 & 537 & 565 & Yes & Tunisia & Saidi et al., 2010 [64] \\
\hline NPPA & $1 \mathrm{p} 12$ & $\begin{array}{c}\text { rs748566461 } \\
\text { C1364A } \\
\text { C55A }\end{array}$ & NA & NA & NA & 298 & 278 & Yes & South Africa & Nkeh et al., 2002 [32] \\
\hline CYP2C8 & $10 q 23.331$ & $\begin{array}{l}\text { rs10509681 } \\
\text { rs11572080 }\end{array}$ & $\begin{array}{l}\mathrm{T}>\mathrm{C} \\
\mathrm{C}>\mathrm{T}\end{array}$ & $\begin{array}{l}0.046 \\
0.046\end{array}$ & $\begin{array}{l}0.008 \\
0.008\end{array}$ & NA & NA & Yes & Ghana & Williams et al., 2004 [43] \\
\hline
\end{tabular}


Table 2. Cont

\begin{tabular}{|c|c|c|c|c|c|c|c|c|c|c|}
\hline Gene & $\begin{array}{c}\text { Chr } \\
\text { Position }\end{array}$ & SNP & Alleles & $\begin{array}{c}\text { Alt Allele Freq, } \\
\text { Global (db. SNP) }\end{array}$ & $\begin{array}{l}\text { Alt Allele Freq, } \\
\text { African (db. SNP) }\end{array}$ & Cases No. & Controls & $\begin{array}{l}\text { Association } \\
(p<0.05)\end{array}$ & Country & Author Year \\
\hline$L E P$ & $7 \mathrm{q} 32.1$ & rs7799039 & $\mathrm{G}>\mathrm{A}$ & 0.402 & 0.032 & 45 & 53 & Yes & Tunisia & Ben et al., 2008 [65] \\
\hline$A D D 1$ & 4 p16.3 & rs4961 & $\mathrm{G}>\mathrm{T}$ & 0.208 & 0.049 & 148 & 94 & No & South Africa & Barlassina et al., 2000 [66] \\
\hline$A D R B 2$ & $5 q 32$ & $\begin{array}{l}\text { rs1042713 } \\
\text { rs1042714 }\end{array}$ & $\begin{array}{l}\mathrm{G}>\mathrm{A} \\
\mathrm{G}>\mathrm{C}\end{array}$ & $\begin{array}{l}0.476 \\
0.796\end{array}$ & $\begin{array}{l}0.520 \\
0.864\end{array}$ & 192 & 123 & No & South Africa & Candy et al., 2000 [66] \\
\hline $\begin{array}{c}\text { SUB1 } \\
\text { CEP83 } \\
\text { IGFBP3 } \\
\text { CHIC2 } \\
\end{array}$ & $\begin{array}{c}5 \mathrm{p} 13.3 \\
12 \mathrm{q} 22 \\
7 \mathrm{p} 12.3 \\
4 \mathrm{q} 12 \\
\end{array}$ & $\begin{array}{c}\mathrm{rs} 7726475 \\
\mathrm{rs} 11837544 \\
\mathrm{rs} 11977526 \\
\mathrm{rs} 11725861 \\
\end{array}$ & $\begin{array}{l}\mathrm{G}>\mathrm{A} \\
\mathrm{T}>\mathrm{A} \\
\mathrm{G}>\mathrm{A} \\
\mathrm{A}>\mathrm{G}\end{array}$ & $\begin{array}{l}0.187 \\
0.081 \\
0.442 \\
0.169 \\
\end{array}$ & $\begin{array}{l}0.024 \\
0.216 \\
0.326 \\
0.181 \\
\end{array}$ & 782 & 2099 & Yes & Uganda & Kayima et al., 2017 [33] \\
\hline AGTR2 & $\mathrm{Xq23}$ & rs11091046 & NA & NA & NA & 382 & 403 & No & Tunisia & Kabadou et al., 2012 [67] \\
\hline CPS1 & $2 q 34$ & rs1047891 & $\mathrm{C}>\mathrm{A}$ & 0.289 & 0.368 & NA & NA & Yes & Ghana & Williams et al., 2004 [43] \\
\hline MOV10 & $1 \mathrm{p} 13.2$ & rs2932538 & $A>C$ & 0.830 & 0.842 & 189 & 598 & Yes & Algeria & Lardjam-Hetraf et al., \\
\hline SLC4A7 & 3p24.1 & rs13082711 & $\mathrm{T}>\mathrm{C}$ & 0.120 & 0.036 & & & & & 2015 [53] \\
\hline MECOM & $3 q 26.2$ & rs419076 & $\mathrm{T}>\mathrm{A}$ & 0.584 & 0.450 & & & & & \\
\hline SLC39A8 & $4 q 24$ & rs13107325 & $\mathrm{C}>\mathrm{A}$ & 0.024 & 0.002 & & & & & \\
\hline GUCY1A1N & $4 \mathrm{q} 32.1$ & rs13139571 & $\mathrm{C}>\mathrm{A}$ & 0.211 & 0.129 & & & & & \\
\hline PR3 & 19p13.3 & rs1173771 & $A>G$ & 0.661 & 0.795 & & & & & \\
\hline HFE & $6 \mathrm{p} 22.2$ & rs1799945 & $\mathrm{C}>\mathrm{G}$ & 0.073 & 0.011 & & & & & \\
\hline CACNB2 & 10p12.33 & rs4373814 & $G>C$ & 0.512 & 0.613 & & & & & \\
\hline PLCE1 & $10 \mathrm{q} 23.33$ & rs932764 & $A>G$ & 0.428 & 0.184 & & & & & \\
\hline CAND1 & $12 q 14$ & rs7129220 & $\mathrm{G}>\mathrm{A}$ & 0.058 & 0.052 & & & & & \\
\hline ARHGAP42 & $11 q 22.1$ & rs633185 & $G>A$ & 0.639 & 0.804 & & & & & \\
\hline FES & 15q26.1 & rs2521501 & $A>C$ & 0.213 & 0.223 & & & & & \\
\hline GOSR2 & $17 q 21.32$ & rs17608766 & $\mathrm{T}>\mathrm{C}$ & 0.054 & 0.010 & & & & & \\
\hline ZNF831 & 20q13.32 & rs6015450 & $A>G$ & 0.098 & 0.205 & & & & & \\
\hline ULK4 & 3p22.1 & rs3774372 & $\mathrm{T}>\mathrm{C}$ & 0.173 & 0.197 & & & & & \\
\hline CABCOCO1 & 10q21.2 & rs4590817 & $G>C$ & 0.110 & 0.190 & & & & & \\
\hline SH2B3 & $12 \mathrm{q} 24.12$ & rs3184504 & $\mathrm{T}>\mathrm{A}$ & 0.853 & 0.981 & & & & & \\
\hline TBX5 & $12 \mathrm{q} 24.21$ & rs10850411 & $\mathrm{T}>\mathrm{C}$ & 0.470 & 0.346 & & & & & \\
\hline CSK & $15 q 24.1$ & rs1378942 & $\mathrm{C}>\mathrm{A}$ & 0.245 & 0.026 & & & & & \\
\hline ZNF652 & $17 q 21.32$ & rs12940887 & $\mathrm{C}>\mathrm{T}$ & 0.185 & 0.048 & & & & & \\
\hline
\end{tabular}


Northern African countries $(n=25)$ appeared to have more studies carried on HTN and SNPs association sub-Saharan African countries (Eastern Africa $=2$, Central Africa $=1$, Western Africa $=6$, Southern Africa $=8$ ) as elaborated in Figure 2. Twenty studies reported an association between HTN and genes such as ACE, AGT, AGTR1, ANP, APOA5, ARGHGAP42, ATP2B1, B2, BAG6, CABCOCO1, CACNB2, CAND1, CHIC2, CNNM2, CPS1, CSK, CYP11B2, CYP2C8, EBF1, FES, FGF5, GNB3, GOSR2, GRK4, GUCY1A1N, HFE, IGFBP3, JAG1, LEP, MECOM, MOV10, MTHFR, NOS3, PLCE1, PLEKHA7, PR3, SH2B3, SLC39A8, SLC4A7, STK39, SUB1, TBX5, ULK4, ZNF652, and ZNF831 whereas twenty-two studies did not show any association (Table 2). The most studied genes were AGT, ACE, NOS3, AGTR1, MTHFR, ATP2B1, CYP2C8, GNB3, CNNM2, PLEKHA7, JAG1, FGF5, and EBF1 as sequentially arranged from highest to the smallest number of studies (see Table 2). Other genes reported in single studies were STK39, CDKAL1, IGF2BP2, TH, B2, CYP11B2, LEP, CLCNKB, SCNN1B, ADD1, ADRB2, SUB1, CEP83, IGFBP3, CHIC2, AGTR2, CPS1, MOV10, SLC4A7, MECOM, SLC39A8, GUCY1A1N, PR3, HFE, BAG6, CACNB2, PLCE1, CAND1, ARHGAP42, FES, GOSR2, ZNF831, ULK4, CABCOCO1, SH2B3, TBX5, CSK, and ZNF652.

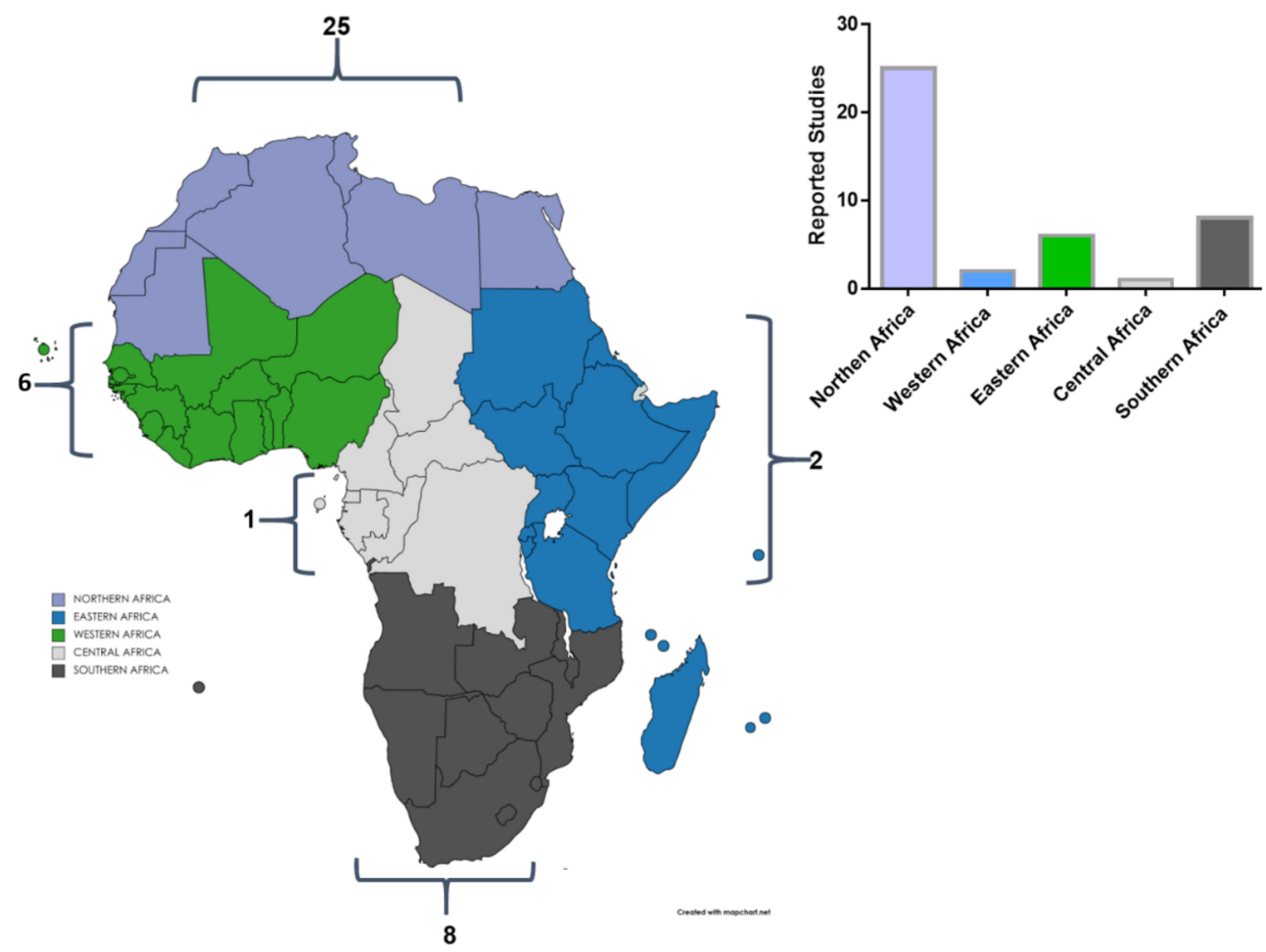

Figure 2. Summary of all genetic studies reported in the African continent in relation to hypertension.

\subsection{Gene-Drug Interaction and Ontology Analysis}

A gene list consisting of 53 genes (Table S1) linked to HTN was analyzed to gain an understanding of drug interaction, biological processes, pathways and various associated diseases Food and Drug Administration (FDA) approved drug list was obtained (Table S2), and used to identify drug interactions with the above-mentioned genes. Identification of potential drug-gene interactions is the first step in a translation research effort aiming to reduce the burden of a major public health problem such as CVD [68]. Therefore, in the current study Drug Bank and CTD databases were utilized to generate a drug-gene interaction network (Figure 3) that identified the genes interacting with FDA approved HTN drugs. Of the 53 genes, only 14 genes (CYP2C8, CYP11B2, AGT, AGTR1, AGTR2, ACE, ADRB2, LEP, MTHFR, NOS3, HFE, CNNM3, IGF2BP2, and SCNN1B) showed an 
interaction with marketed drugs along with their corresponding side-effects (Figure 3). GO enrichment analysis was performed to identify the enriched biological processes that the gene list regulates (Figure 4). Finding ontologies linked to biological processes provides insights into the underlying mechanisms regulated by the chosen gene list. This allows for the identification of additional drugs that have previously not treated HTN.

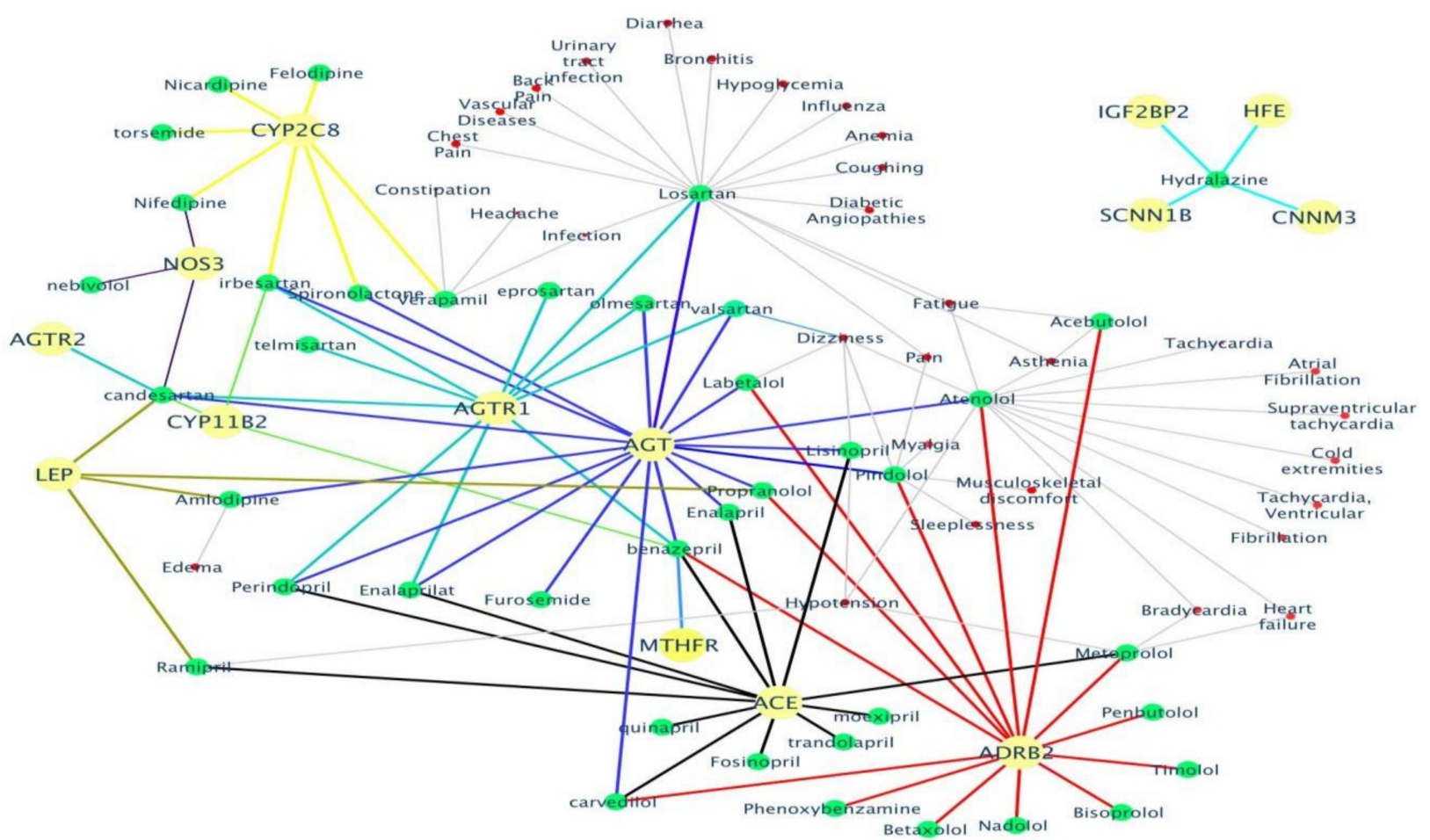

Figure 3. The identified drug-gene interactions along with the side-effects are plotted into a network. Only 14 out of the total 53 HTN genes (shown as yellow nodes) mapped to 57 FDA approved HTN drugs (shown as green nodes) and their corresponding side-effects (shown as red nodes). The edges in the drug-gene interaction network are shown in a different color for each gene to easily distinguish their association with the various HTN drugs.

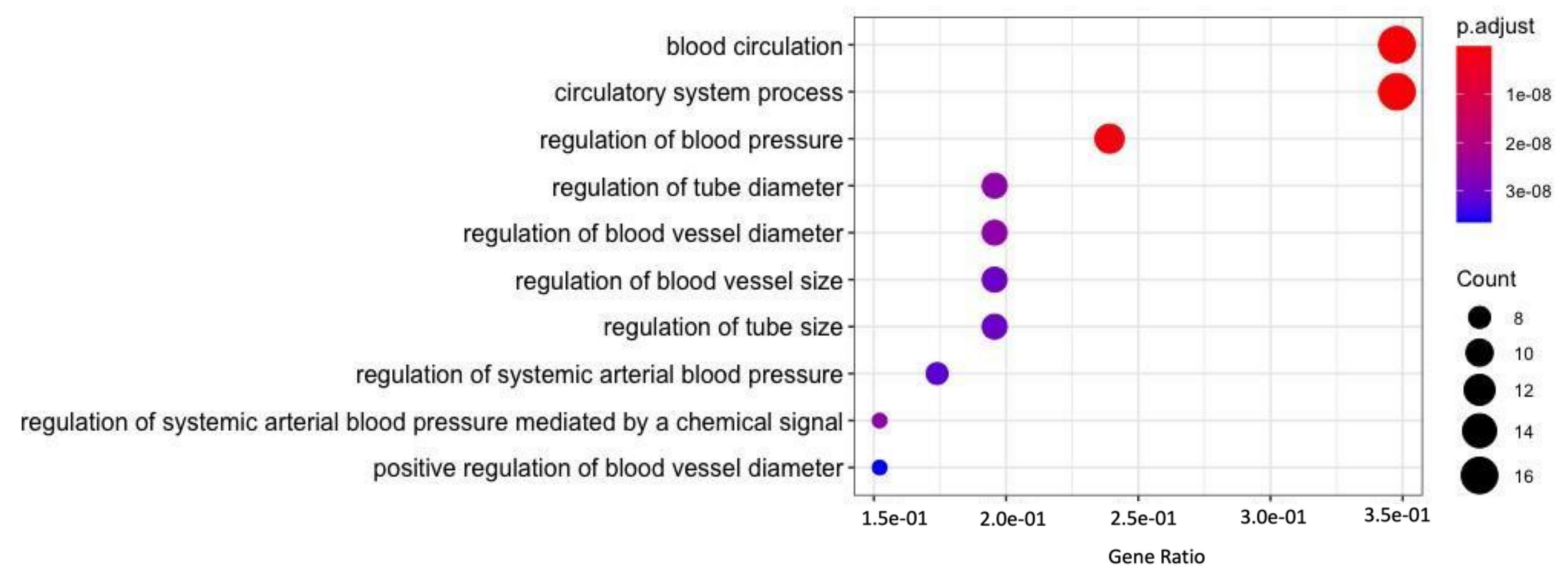

Figure 4. The gene ontology analysis performed on all 53 prioritized HTN related genes to identify related biological processes. The gene ratio of participating genes in the enriched ontology, the color-coded Benjamini-Hochberg (BH)-adjusted $p$-value, and the number of genes (count) in each enriched ontology are shown in the above plot.

Co-expression analysis was performed to identify co-expressed gene clusters (Figure 5). These were interrogated further to relate the molecular function for each gene cluster (Figure 6). This was performed to identify potential mechanisms in which drugs can be 
targeted against HTN. Genes in co-expressed gene clusters and their linked HTN drugs are summarized in Table S4 to explore potential combination therapy options.

Cluster 1

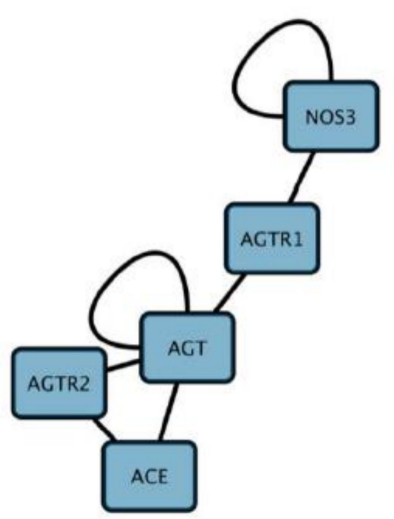

Cluster 2

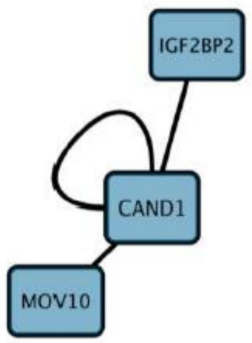

Cluster 3

Figure 5. Co-expression networks generated to identify co-regulated gene clusters from the 53 prioritized genes related to Hypertension Co-expressed gene clusters have been numbered above from 1 to 3 .

oxidoreductase activity, acting on $\mathrm{NAD}(\mathrm{P}) \mathrm{H}, \mathrm{H}$ G protein-coupled receptor binding
G protein-coupled peptide receptor activity
peptide receptor activity
dipeptidyl-peptidase activity mitogen-activated protein kinase kinase binding TBP-class protein binding mRNA 5'-UTR binding

ATP-dependent RNA helicase activity RNA-dependent ATPase activity RNA helicase activity translation regulator activity ATP-dependent helicase activity adrenergic receptor activity adenylate cyclase binding protein kinase $A$ catalytic subunit binding catecholamine binding proline-rich region binding $\mathrm{G}$ protein-coupled amine receptor activity non-membrane spanning protein tyrosine kinase activity

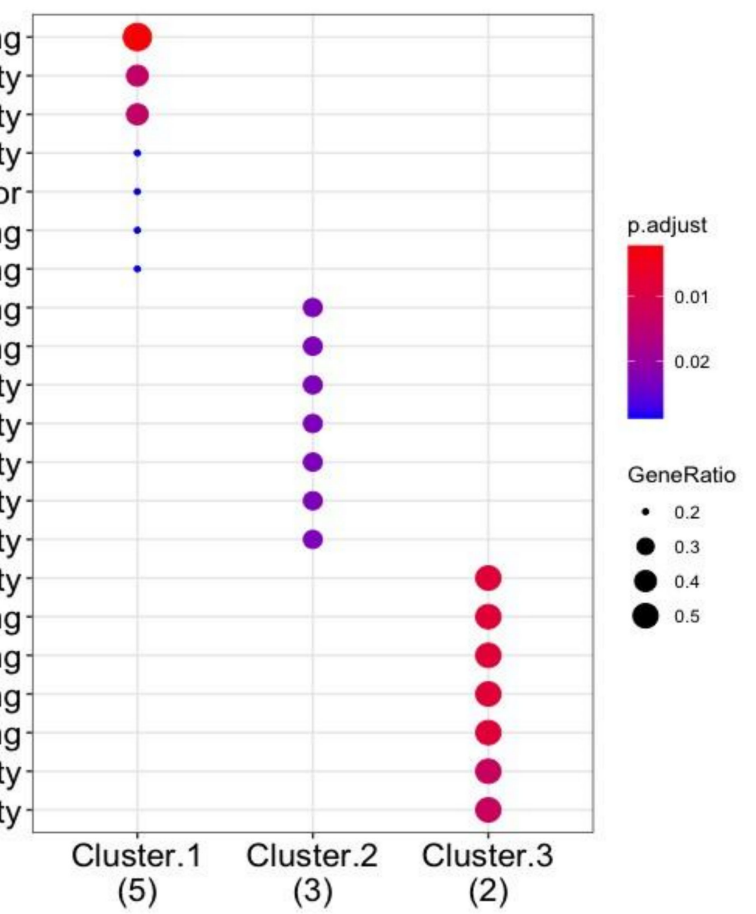

Figure 6. GO enrichment (i.e., molecular function) performed on the co-expressed gene clusters (labelled cluster 1-3, and the number of genes in brackets) to relate molecular function to co-expressed genes. The gene ratio of participating genes in the enriched ontology, and the color coded BH-adjusted $p$-value are shown in the plot.

\subsection{Co-Expression Ontology Analysis}

Similar to the GO enrichment analysis performed, KEGG pathway analysis (Figure 7) was performed to identify the enriched pathways regulated by the $53 \mathrm{HTN}$ genes. This is performed to identify alternative pathways that interact with HTN related pathways, which can provide insights into how HTN drugs may influence various biological pathways. 


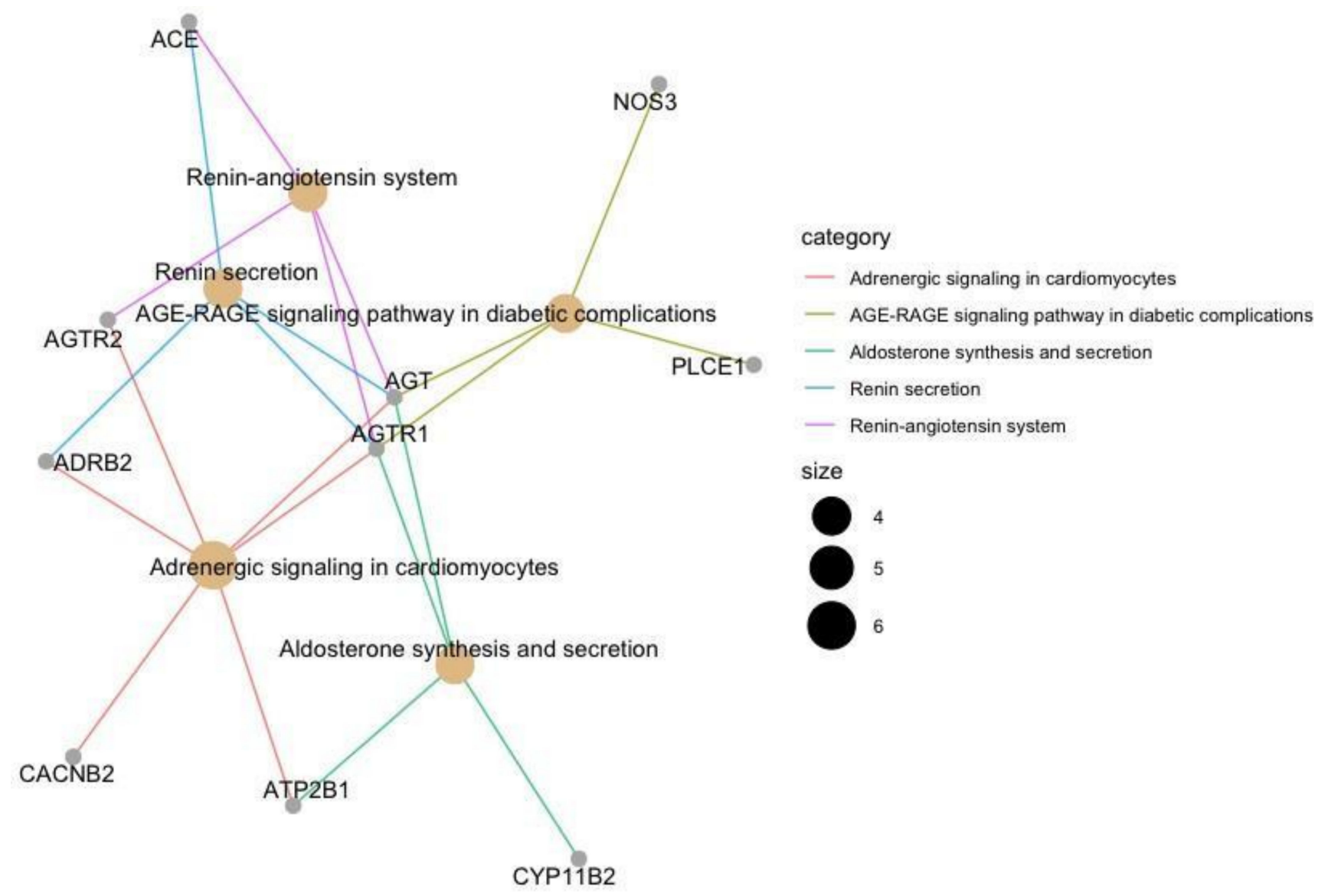

Figure 7. Pathway enrichment analysis performed on the 53 HTN genes annotated using the KEGG database. Only 10 from the 53 HTN genes mapped to the KEGG database linking them to biological pathways. The plot illustrates the participating genes with the enriched pathway. Each enriched pathway has been color-coded, and the number of participating genes corresponds to the size of the node.

\subsection{Biological Pathway Analysis}

Disease ontology (Figure 8) was also performed to analyze the involvement of the 53 HTN genes in various other diseases. This analysis gives insights into how different diseases may share common gene expression patterns and how drug treatment influences several biological pathways causing undesired side effects. In the selection process of identifying genes that are only linked to hypertension and other related diseases, DO analysis was conducted.

\subsection{Disease Ontology}

Disease ontology built from a disease-gene interaction database was plotted in a network (Figure 9) to visualize the interactions between genes and enriched diseases. This graph serves to identify genes that are targeted in HTN treatment however are also linked with other diseases. 


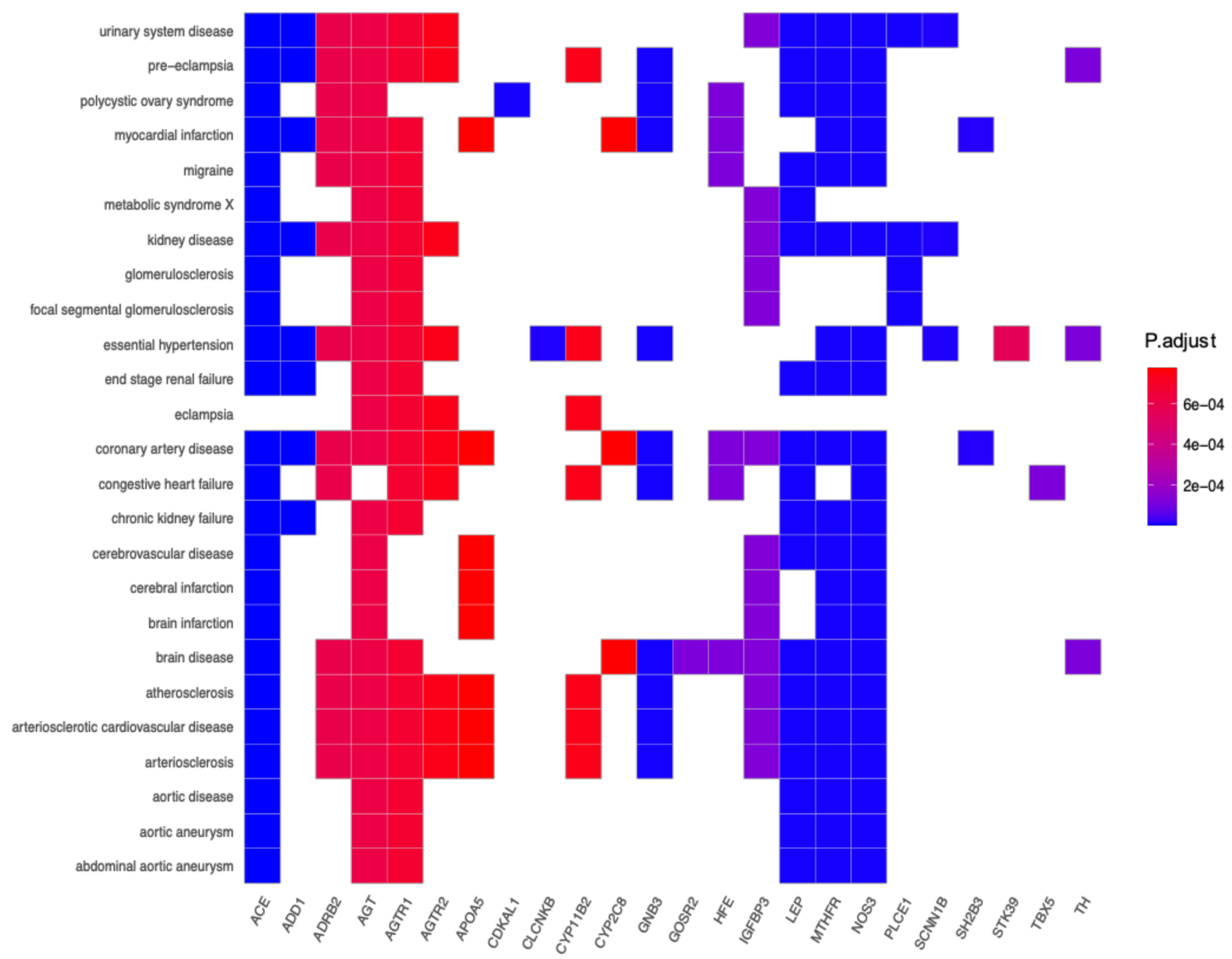

Figure 8. The enrichment of disease ontology for the mapped genes related to hypertension. Each gene is statistically mapped to a disease. The matched boxes associated with the enriched disease term are color coded by the BH-adjusted $p$-values.

\section{Discussion}

HTN is a multifactorial disease affecting one billion individuals. It is a leading cardiovascular risk factor accounting for premature deaths and has a significant economic cost [15]. Despite the availability of many antihypertensive drugs, less than $50 \%$ of patients have their blood pressure controlled [69]. These disappointing outcomes could be because of medication non-adherence and/or interindividual variation [4]. Considering studies linking interindividual variation to drug response, several studies have tested SNPs as predictors of TRH risk [70-72]. Various studies have reported an association between SNPs and HTN in European [73,74], African American [75], and Asian population [75-77], however not much evidence has been generated on the association of specific SNP or its associated haplotypes with HTN in an African population [27]. Therefore, we systematically extracted and discussed evidence on the African genetic variation and pharmacogenomics towards the treatment of HTN. 


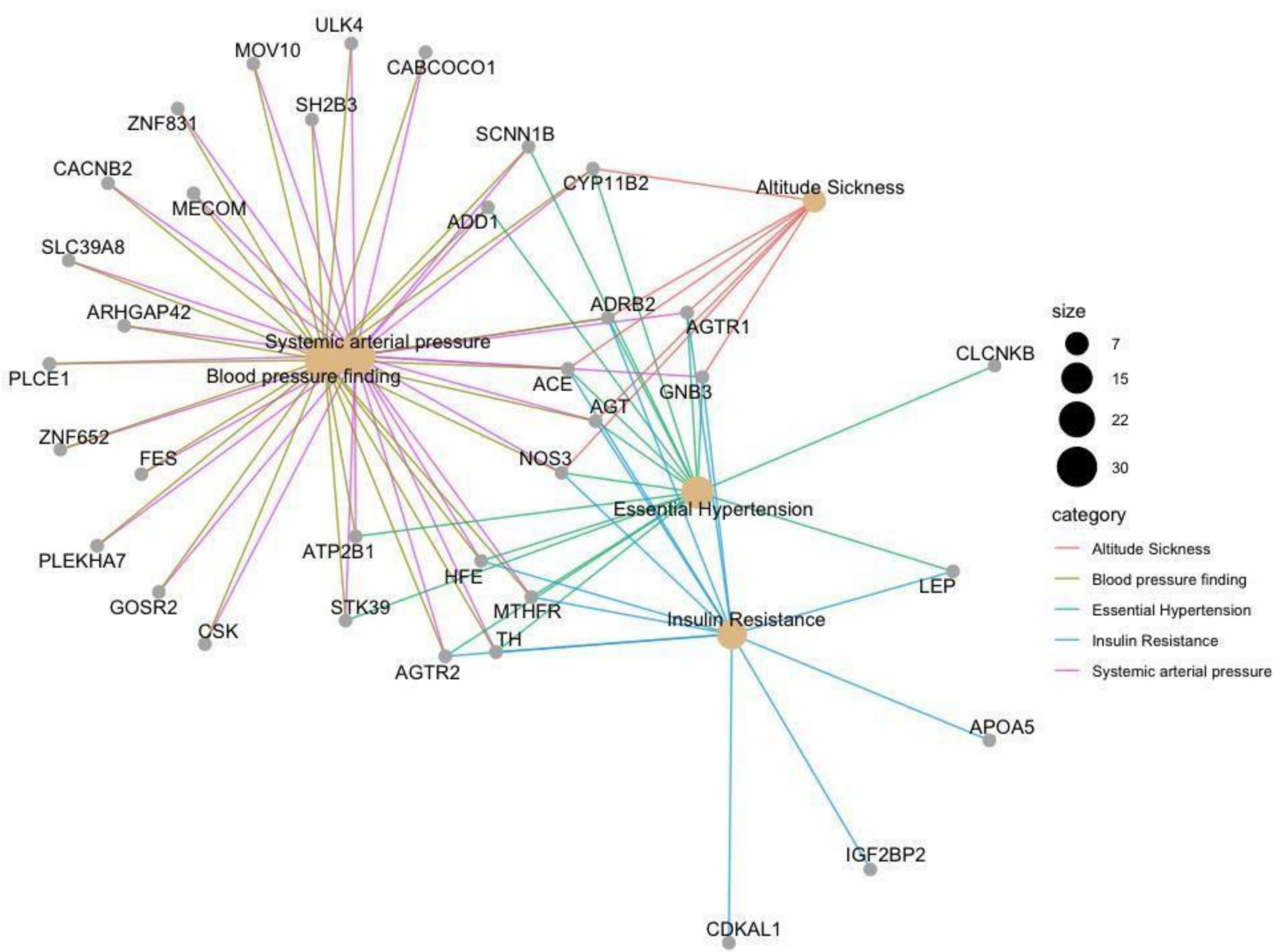

Figure 9. The gene-disease interaction network of the mapped 53 genes related to HTN to the disease ontology database. The size of the enriched disease illustrated as a node corresponds to the number of participating genes. The color of the edges corresponds to the enriched disease.

A total of 42 studies comprising of 53 genes are included in this review, however, only 20 studies reported a significant association with the risk of HTN. Table 2 highlighted candidate genes such as ACE, NOS3, ATP2B1, and MTHFR that have repeatedly been implicated to have an association with HTN in various African populations.

\subsection{Angiotensin-Converting Enzyme Gene}

Angiotensin-converting enzyme $(A C E)$ is a metalloenzyme that cleaves angiotensin I to angiotensin II and inactivates a potent vasodilator [78]. ACE is encoded by the ACE gene which has been mapped to chromosome 17q23, and it has 26 exons and 25 introns [44]. Single-nucleotide polymorphism (rs1799752) on the ACE gene was investigated for its implication on high blood pressure in different sub-Saharan Africa countries (Table 2). It has been showed in various studies conducted in different parts of Africa continent that rs1799752 could be a potential genetic predictor for the development of HTN and permit initiation of personalized medicine [76-78]. However, other studies performed in South Africa, Tunisia, and Egypt exhibited no association of $A C E$ gene polymorphism with the risk of developing HTN [38,40,79].

\subsection{Nitric Oxide Synthase Gene}

Nitric oxide synthase (NOS3) gene is another important candidate gene because of its critical role in regulating blood pressure. This gene (NOS3) is on chromosome 7q35-36, 
comprising 26 exons. NOS3 is an integral component of vasorelaxing pathway, which is mediated by endothelium derived nitric oxide [80]. Various studies summarized in this review showed that different populations from Africa exhibit positive association of genetic variants of NOS3 such as rs1799983 [47], rs2070744 [49], rs149868979 [58] and rs61722009 [51] with the risk of developing HTN. However, two studies in this review that aimed to investigate the NOS3 gene variants associated with the development of HTN showed no association with the disease $[48,50]$.

\subsection{Plasma Membrane Calcium-Transporting ATPase 1 Gene}

In 2009, the genome-wide association studies (GWAS) have identified the association between the Plasma membrane calcium-transporting ATPase 1 (ATP2B1) polymorphisms (rs2681472, rs17249754, rs2681492) and HTN in diverse populations [81,82]. This gene encodes for plasma membrane calcium dependent ATPase, which handles calcium pumping to the extracellular compartment [83]. In this review, it was revealed that genetic variants in ATP2B1 genes in some populations from Algeria, Burkina Faso, and Uganda are associated with the risk of developing HTN. For instance, a study conducted in an Algerian population reported that the ATP2B1 variant (rs17249754) has a robust significant association with HTN [53]. The findings were further replicated in the Burkinabe population [3]. In another study conducted by Kayima et al. [33] on a Ugandan population, it was reported that rs2681492 was significantly associated with HTN in hypertensive individuals. Likewise, SNPs on ATP2B1 (rs17249754, rs2681472, and rs2681492) were reported to be in linkage disequilibrium and located in the same linkage disequilibrium block in the Chinese population [84].

\subsection{Methylenetetrahydrofolate Reductase Gene}

Methylenetetrahydrofolate reductase gene (MTHFR) is one of the most studied genes associated with HTN in the African countries. MTHFR is on chromosome 1p36.3 and is well known to be involved in the metabolism of homocysteine and folate [85]. Previous studies have shown that the MTHFR variant such as rs1801133 has been associated with HTN between $24-87 \%$ [86-88]. Similarly, in this review, $50 \%$ of studies investigating the role of MTHFR provided a positive line of evidence linking this gene with HTN, showing that the rs1801133 polymorphism in MTHFR increases the risk of HTN $[53,56]$. Conversely, Amrani-Midoun et al. [47] and Amin et al. [55] in Algeria and Egypt showed no association of rs1801133 in hypertensive patients.

Furthermore, genes such as CYP11B2 (rs1799998) [64], LEP (rs7799039) [65], and CPS1 (rs1047891) [89] were also reported to be associated with HTN, however, these findings could not be replicated in the different African populations. As aforementioned, $A C E$ and NOS3 genes have been studied for its implication on HTN in more than six published papers indexed on the different databases, of which $50 \%$ of those were able to link these genes polymorphisms with the high risk for HTN. This suggests that there was a significant association of $A C E$ and NOS3 variants with HTN in African populations. However, AGT, AGTR1, and AGTR2 polymorphisms in African populations showed less than $25 \%$ or no association with HTN. For example, all the SNPs located near AGT gene, only rs2004776 was demonstrated to have a strong association with HTN in Ugandan population [81]. In a recent study by EI-Garawani et al. [90], two polymorphisms (rs4762 and rs699) of $A G T$ gene were found to be associated with the risk of diabetes and hypertension in the Egyptian population. Furthermore, Farrag et al. [42] reported the positive association of AGTR1, rs5186 with HTN in Egyptian population and similar findings were also observed in the Ghanaian population. However, no association has been recorded in other AGTR1 and AGTR2 polymorphisms.

\subsection{Computational Insights}

Furthermore, we attempted to shortlist key target genes out of the 53 identified genes in this review by using bioinformatics methods on gene-specific data available in various 
public databases. The bioinformatics analysis investigated various parameters associated with the 53 HTN genes such as drug interactions, co-expression of genes and disease ontology, and linked pathways. Only 14 genes (CYP2C8, CYP11B2, AGT, AGTR1, AGTR2, $A C E, A D R B 2, L E P, M T H F R$, NOS3, HFE, CNNM3, IGF2BP2, and SCNN1B) were identified to have an interaction with FDA approved marketed HTN drugs (Figure 3 and Table S4). As evident from Figure 3, the various antihypertensive drugs such as losartan, acebutolol, atenolol, metoprolol, ramipril, lisinopril, labetalol, and amlodipine show distinct side-effects. It has also been reported in the literature [91-93] that $20-97 \%$ of patients taking antihypertensive drugs suffer from various drug-related side effects. Furthermore, these side effects may potentially also account for non-adherence to antihypertensive drugs [94-96]. Losartan, an angiotensin II antagonist and atenolol, a $\beta$-blocker showed the most side-effects related to respiratory impairments and infection. Prior studies have also documented a higher frequency of side-effects and lower adherence among patients taking diuretics and $\beta$-blockers [97]. In hypertensive patients of African ancestry, diuretics and or calcium channel blockers (amlodipine) are preferably recommended [98] and patients who remain concerned about the adverse health effects of antihypertensive drugs are less likely to be adherent to their medications [99].

The co-expression analysis identified three clusters, including cluster 1 (ACE, AGT, AGTR1, AGTR2, and NOS3), cluster 2 (MOV10, CAN1, and IGF2BP2), and cluster 3 (CSK and ADRB2). Upon further analysis, from cluster 1, AGTR1, AGTR2, AGT, and ACE; cluster 2, IGFBP2 and cluster $3 A D R B 2$ genes are shown to be mapped to HTN drugs shown in Figure 3 and Table S4. Interestingly, the protein-coding genes AGTR1, AGTR2, AGT, and $A C E$ are all involved in the RAAS cascade which regulates blood pressure and vascular resistance. As such, the most widely used HTN drug classes include ACE inhibitors, angiotensin II antagonists and direct renin inhibitors which target the RAAS cascade [100]. Whereas, in the second and third cluster, the genes IGFBP2 (targeted by vasodilators) and $A D R B 2$ (targeted by $\beta$-blockers), respectively, have been recently linked with increased risk of HTN and have been recently proposed as potential diagnostic biomarkers for HTN. For example, in a study by Yang et al. [101] the overexpression of the gene IGFBP2 which is linked to the regulation of vascularization and angiogenesis is associated with the increased severity of HTN. Additionally, the gene ADRB2 has been shown to interact with the gene NOS3 [102]. ADRB2 may indirectly interact with the RAAS cascade via the NOS3 forming part of the gene cluster of genes associated with the RAAS cascade (Figure 5). This is further illustrated in Figure 7 where ADRB2 shows interaction with RAAS associated genes by regulating common biological pathways including renin secretion and adrenergic signaling. Lastly, the observations in Figures 7-9 highlight the genes ADRB2, ACE, AGT, and NOS3 to have an association with the metabolic syndrome (includes hypertension, type II diabetes, excess lipids and abnormal cholesterol levels). Although further validation is required, the above-mentioned genes exhibit characteristics of being key regulators in metabolic syndrome.

This is also evident from the biological processes and pathways showed enrichment of HTN (by vasoconstriction and RAAS) and diabetic-related terms (see Figures 6 and 7). NOS3 (mapped to drug-gene interaction network) and PLCE1 showed no interaction with HTN related KEGG pathways but exclusive to AGE-RAGE signaling pathways in diabetic complications. As such, using drugs targeted against HTN may influence other signaling pathways. In this case, NOS3 interacts with 3 HTN drugs (Angiotensin II antagonists, calcium channel blockers, and $\beta$-blockers).

It is also important to understand the roles that genes play in different sub-types of HTN. Figure 8 shows that certain genes are shared among different sub-types of HTN and there are genes which contribute to a specific sub-type of the disease. For example, $C L C N K B$ is only linked to $T R H$, therefore, a drug targeting this gene will be effective in treating salt sensitive HTN, since a polymorphism in this gene leads to increased salt retention in the bloodstream. Similarly, the gene such as $A C E$ is linked to almost all categories of diseases shown in Figure 8, thus pointing towards its universal role in HTN related diseases. 
From the DO analysis, the following potential gene targets have been selected, these include CLCNKB, CYPB11B2, SH2B2, STK9, and TBX5 as they show interactions exclusively with hypertension-related pathways (see Figure 8). Furthermore, targeting co-regulated gene clusters as opposed to single-gene targeting increases the likelihood of more effective drug treatment for HTN. Cluster 1 (ACE, AGT, AGTR1, AGTR2, and NOS3) and cluster 3 (CSK and ADRG1) showed enrichment of G-coupled receptor binding activity (a classical target of drug discovery) and protein tyrosine kinase receptors (see Figure 5), thus demonstrates that these gene clusters can be targeted during future drug design. Gene-gene interactions among regulatory variants play a critical role in the drug-response phenotype $[103,104]$. Based on the previous research, and findings in the current research paper it has been suggested that all genes within a cluster should be screened and molecular mechanisms supporting various gene-gene interactions should be explored that may enable identification of potential pharmacogenomic biomarkers to guide personalized medicine approaches for HTN. Therefore, we anticipate that these potential genes including their SNPs need to be investigated further in African populations.

While several studies in this review support an important contribution of genetic polymorphisms in HTN, some studies have failed to detect significant effects or replicate previous findings. These inconsistencies may be linked to the African population's high genetic diversity and low levels of linkage disequilibrium among loci when compared to populations from other countries outside Africa [105]. Furthermore, studies suggest that from a genetic standpoint, there is no SNP-database that can be used to personalize treatments for HTN in an African population $[47,106,107]$. Inferring that the lack of genetic information with robust allele frequency distributions serves as a hurdle to implement corrective treatment and this may have important medical implications [108]. Providing a more accurate reference foundation on which to support future disease research in Africa is thus of utmost importance [27].

\section{Conclusions}

In conclusion, the present study reported on the susceptibility of inter-individual genetic variation to HTN in African population. Large-scale genetic studies are needed to better understand the susceptibility of African population-based inter-individual genetic variation and their effect on the hypertensive drug response, which will aid in the development of effective African based individualized antihypertensive medicine.

Supplementary Materials: The following are available online at https:/ / www.mdpi.com/article/ 10.3390/genes12040532/s1, Table S1: Publication Search, Table S2: The list of the prioritized 53 genes used for the in silico analysis, Table S3: The list of FDA drugs and their grouped according to their action mechanism, Table S4: Drugs associated with genes that are part of the co-expressed gene clusters.

Author Contributions: S.E.M. conducted systematic literature search and collected and analyzed the data and compiled the first draft of paper. L.M. and M.K. performed bioinformatics analysis and reviewed and edited the manuscript. J.R.S. contributed to design, analysis and edited the manuscript. T.A. reviewed and edited the manuscript. B.M. contributed in design, analysis of the manuscript. M.B. reviewed and edited the manuscript. R.J. conceptualized, designed, reviewed and edited the manuscript. All authors have read and agreed to the published version of the manuscript.

Funding: This research was funded by the South Africa Medical Research Council's SHIP-RFA-012019 Strategic Health Innovation Partnership (Pharmacogenomics in Precision Medicine), Biomedical Research and Innovation Platform baseline funding as well as Division of Research Capacity Development Internship Scholarship Program (RCD), the National Research Foundation (NRF) Competitive Program for Rated Researchers (UID120812) and NRF bursary (No: MND190721457781).

Institutional Review Board Statement: Not applicable.

Informed Consent Statement: Not applicable.

Data Availability Statement: Not applicable. 
Acknowledgments: The work reported herein was made possible through funding by the South Africa Medical Research Council's SHIP-RFA-01-2019 Strategic Health Innovation Partnership (Pharmacogenomics in Precision Medicine), Biomedical Research and Innovation Platform baseline funding as well as Division of Research Capacity Development Internship Scholarship Program (RCD), and the National Research Foundation (NRF) Competitive Program for Rated Researchers (UID120812). NRF bursary (No: MND190721457781) to L.M. is also acknowledged.

Conflicts of Interest: The authors declare that they have no conflict of interest.

\section{References}

1. Owiredu, W.K.B.A.; Appiah, M.; Obirikorang, C.; Adu, E.A.; Boima, V.; Amos-Abanyie, E.K.; Akyaw, P.A.; Owiredu, E.-W.; Acheampong, E. Association of MYH9-rs3752462 polymorphisms with chronic kidney disease among clinically diagnosed hypertensive patients: A case-control study in a Ghanaian population. Clin. Hypertens. 2020, 26, 1-9. [CrossRef]

2. Bloch, M.J. Worldwide prevalence of hypertension exceeds 1.3 billion. J. Am. Soc. Hypertens. 2016, 10, 753-754. [CrossRef] [PubMed]

3. Sombié, H.K.; Kologo, J.K.; Tchelougou, D.; Ouédraogo, S.Y.; Ouattara, A.K.; Compaoré, T.R.; Nagalo, B.M.; Sorgho, A.P.; Nagabila, I.; Soubeïga, S.T.; et al. Positive association between ATP2B1 rs17249754 and essential hypertension: A case-control study in Burkina Faso, West Africa. BMC Cardiovasc. Disord. 2019, 19, 155. [CrossRef] [PubMed]

4. Zhou, B.; Bentham, J.; Di Cesare, M.; Bixby, H.; Danaei, G.; Cowan, M.J.; Paciorek, C.J.; Singh, G.; Hajifathalian, K.; Bennett, J.E.; et al. Worldwide trends in blood pressure from 1975 to 2015: A pooled analysis of 1479 population-based measurement studies with $19 \cdot 1$ million participants. Lancet 2017, 389, 37-55. [CrossRef]

5. Cheong, A.T.; Sazlina, S.G.; Tong, S.F.; Azah, A.S.; Salmiah, S. Poor blood pressure control and its associated factors among older people with hypertension: A cross-sectional study in six public primary care clinics in Malaysia. Malays. Fam. Phys. 2015, 10, 19-25.

6. Firmo, J.O.A.; Mambrini, J.V.d.M.; Peixoto, S.V.; Loyola Filho, A.I.d.; Souza Junior, P.R.B.d.; Andrade, F.B.d.; Lima-Costa, M.F. Adequate control of hypertension among older adults: ELSI-Brazil. Revista Saude Publica 2018, 52, 13s. [CrossRef]

7. Pender, K.; Omole, O. Blood pressure control and burden of treatment in South African primary healthcare: A cross-sectional study. Afr. J. Prim. Health Care Fam. Med. 2019, 11, 7. [CrossRef]

8. Seedat, Y.; Rayner, B.; Veriava, Y. South African hypertension practice guideline 2014. Cardiovasc. J. Afr. 2014, 25, 288-294. [CrossRef]

9. Yaxley, J.P.; Thambar, S.V. Resistant hypertension: An approach to management in primary care. J. Fam. Med. Prim. Care 2015, 4, 193-199. [CrossRef]

10. Mehanna, M.; Gong, Y.; McDonough, C.W.; Beitelshees, A.L.; Gums, J.G.; Chapman, A.B.; Schwartz, G.L.; Johnson, J.A.; Turner, S.T.; Cooper-DeHoff, R.M. Blood pressure response to metoprolol and chlorthalidone in European and African Americans with hypertension. J. Clin. Hypertens. 2017, 19, 1301-1308. [CrossRef]

11. Muntner, P.; Carey, R.M.; Gidding, S.; Jones, D.W.; Taler, S.J.; Wright, J.T.; Whelton, P.K. Potential U.S. Population Impact of the 2017 ACC/AHA High Blood Pressure Guideline. J. Am. Coll. Cardiol. 2018, 71, 109-118. [CrossRef]

12. Musini, V.M.; Gueyffier, F.; Puil, L.; Salzwedel, D.M.; Wright, J.M. Pharmacotherapy for hypertension in adults aged 18 to 59 years. Cochrane Database Syst. Rev. 2017, 8, CD008276. [CrossRef]

13. Pich, J. Pharmacotherapy for hypertension in adults aged 18 to 59 years. Int. J. Nurs. Pr. 2020, 26, e12769. [CrossRef]

14. Carey, R.M.; Whelton, P.K. Prevention, Detection, Evaluation, and Management of High Blood Pressure in Adults: Synopsis of the 2017 American College of Cardiology/American Heart Association Hypertension Guideline. Ann. Intern. Med. 2018, 168, 351-358. [CrossRef]

15. James, P.A.; Oparil, S.; Carter, B.L.; Cushman, W.C.; Dennison-Himmelfarb, C.; Handler, J.; Daniel, T.; Michael, L.; Thomas, D.; Ogedegbe, O.; et al. 2014 evidence-based guideline for the management of high blood pressure in adults: Report from the panel members appointed to the Eighth Joint National Committee (JNC 8). JAMA 2014, 311, 507-520. [CrossRef]

16. Badiu, C. Textbook of Nephro-Endocrinology. Acta Endocrinol. 2017, 13, 523. [CrossRef]

17. Yang, X.; Sood, S.; Luo, Z.; Todd, J.; Wang, J. Genome-Wide Association Studies Identified Resistance Loci to Orange Rust and Yellow Leaf Virus Diseases in Sugarcane (Saccharum spp.). Phytopathol. 2019, 109, 623-631. [CrossRef] [PubMed]

18. Cooper-DeHoff, R.M.; Johnson, J.A. Hypertension pharmacogenomics: In search of personalized treatment approaches. Nat. Rev. Nephrol. 2016, 12, 110-122. [CrossRef] [PubMed]

19. Lauschke, V.M.; Zhou, Y.; Ingelman-Sundberg, M. Novel genetic and epigenetic factors of importance for inter-individual differences in drug disposition, response and toxicity. Pharmacol. Ther. 2019, 197, 122-152. [CrossRef]

20. Cunningham, P.N.; Chapman, A.B. The future of pharmacogenetics in the treatment of hypertension. Future Med. 2019, 20, 129-132. [CrossRef]

21. Ma, Y.; Jiang, H.; Shah, S.J.; Arnett, D.; Irvin, M.R.; Luo, Y. Genetic-Based Hypertension Subtype Identification Using Informative SNPs. Genes 2020, 11, 1265. [CrossRef] [PubMed]

22. Guo, C.; Pei, Q.I.; Tan, H.; Huang, Z.; Yuan, H.; Yang, G. Effects of genetic factors on the pharmacokinetics and pharmacodynamics of amlodipine in primary hypertensive patients. Biomed. Rep. 2015, 3, 195-200. [CrossRef] 
23. Choi, H.D.; Suh, J.H.; Lee, J.Y.; Bae, S.K.; Kang, H.E.; Lee, M.G.; Shin, W.G. Effects of ACE and ADD1 gene polymorphisms on blood pressure response to hydrochlorothiazide: A meta-analysis. Int. J. Clin. Pharmacol. Ther. 2013, 51, 718-724. [CrossRef]

24. Paschos, G.K.; Baggs, J.E.; HogenEsch, J.B.; Fitzgerald, G.A. The Role of Clock Genes in Pharmacology. Annu. Rev. Pharmacol. Toxicol. 2010, 50, 187-214. [CrossRef] [PubMed]

25. Nassereddine, S.; Idrissi, H.H.; Habbal, R.; Abouelfath, R.; Korch, F.; Haraka, M.; Karkar, A.; Nadifi, S. The polymorphism G894 $\mathrm{T}$ of endothelial nitric oxide synthase (eNOS) gene is associated with susceptibility to essential hypertension (EH) in Morocco. BMC Med Genet. 2018, 19, 127. [CrossRef]

26. Raguema, N.; Gannoun, M.; Zitouni, H.; Meddeb, S.; Benletaifa, D.; Lavoie, J.; Almawi, W.; Mahjoub, T. Interleukin-10 rs1800871 $(-819 \mathrm{C} / \mathrm{T})$ and ATA haplotype are associated with preeclampsia in a Tunisian population. Pregnancy Hypertens. 2018, 11, 105-110. [CrossRef]

27. Yako, Y.Y.; Balti, E.V.; Matsha, T.E.; Dzudie, A.; Kruger, D.; Sobngwi, E.; Agyemang, C.; Kengne, A.P. Genetic factors contributing to hypertension in African-based populations: A systematic review and meta-analysis. J. Clin. Hypertens. 2018, 20, 485-495. [CrossRef]

28. Moher, D.; Liberati, A.; Tetzlaff, J.; Altman, D.G. Preferred Reporting Items for Systematic Reviews and Meta-Analyses: The PRISMA Statement. Ann. Intern. Med. 2009, 151, 264-269. [CrossRef]

29. De Bodt, S.; Hollunder, J.; Nelissen, H.; Meulemeester, N.; Inzé, D. CORNET 2.0: Integrating plant coexpression, protein-protein interactions, regulatory interactions, gene associations and functional annotations. New Phytol. 2012, 195, 707-720. [CrossRef]

30. Yu, G.; Wang, L.-G.; Han, Y.; He, Q.-Y. clusterProfiler: An R Package for Comparing Biological Themes Among Gene Clusters. OMICS J. Integr. Biol. 2012, 16, 284-287. [CrossRef]

31. Kabadou, I.; Soualmia, H.; Jemaa, R.; Feki, M.; Kallel, A.; Souheil, O.; Taieb, S.; Sanhaji, H.; Kaabachi, N. G Protein $\beta 3$ Subunit Gene C825T and Angiotensin Converting Enzyme Gene Insertion/Deletion Polymorphisms in Hypertensive Tunisian Population. Clin. Lab. 2013, 59, 85-92. [CrossRef] [PubMed]

32. Nkeh, B.; Tiago, A.; Candy, G.P.; Woodiwiss, A.J.; Badenhorst, D.; Luker, F.; Netjhardt, M.; Brooksbank, R.; Libhaber, C.; Sareli, P.; et al. Association between an atrial natriuretic peptide gene polymorphism and normal blood pressure in subjects of African ancestry. Cardiovasc. J. S. Afr. 2002, 13, 97-101. [PubMed]

33. Kayima, J.; Liang, J.; Natanzon, Y.; Nankabirwa, J.; Ssinabulya, I.; Nakibuuka, J.; Katamba, A.; Mayanja-Kizza, H.; Miron, A.; Li, C.; et al. Association of genetic variation with blood pressure traits among East Africans. Clin. Genet. 2017, 92, 487-494. [CrossRef]

34. Amrani, A.; Hamed, M.B.B.; Talebbendiab, F.M. Association study between some renin-angiotensin system gene variants and essential hypertension in a sample of Algerian population: Case control study. Ann. Biol. Clin. 2015, 73, 557-563. [CrossRef]

35. Tchelougou, D.; Kologo, J.K.; Karou, S.D.; Yaméogo, V.N.; Bisseye, C.; Djigma, F.W.; Ouermi, D.; Compaoré, T.R.; Assih, M.; Pietra, V.; et al. Renin-Angiotensin System Genes Polymorphisms and Essential Hypertension in Burkina Faso, West Africa. Int. J. Hypertens. 2015. [CrossRef]

36. Kooffreh, M.E.; Anumudu, C.I.; Kumar, P.L. Insertion/deletion polymorphism of the angiotensin-converting enzyme gene and the risk of hypertension among residents of two cities, South-South Nigeria. Adv. Biomed. Res. 2014, 3, 118. [CrossRef]

37. Meroufel, D.N.; Mediene-Benchekor, S.; Dumont, J.; Benhamamouch, S.; Amouyel, P.; Brousseau, T. A study on the polymorphisms of the renin-angiotensin system pathway genes for their effect on blood pressure levels in males from Algeria. J. Renin-AngiotensinAldosterone Syst. 2013, 15, 1-6. [CrossRef]

38. Abdraboh, N.R.; Hem, N.S.E.D.; Louka, M.L.; El Bakry, S. Association of Angiotensin Converting Enzyme Insertion/Deletion and Angiotensinogen T235 Polymorphisms with Risk of Essential Hypertension in Egyptian Patients. Int. J. Cancer Res. 2012, 8, 69-82. [CrossRef]

39. Alrefai, A.A.; Habib, M.S.E.-D.; Yaseen, R.I.; Gabr, M.K.; Habeeb, R.M. Association of endothelial nitric oxide synthase (eNOS) gene G894T polymorphism with hypertension risk and complications. Mol. Cell. Biochem. 2016, 421, 103-110. [CrossRef]

40. Ranjith, N.; Pegoraro, R.J.; Rom, L.; Lanning, P.A.; Naidoo, D.P. Renin-angiotensin system and associated gene polymorphisms in myocardial infarction in young South African Indians. Cardiovasc. J. S. Afr. 2004, 15, 22-26.

41. Ghogomu, S.M.; Atanga, R.; Mungwa, S.T.; Muliom, R.N. Lack of association of the A1166C polymorphism in the angiotensin II type 1 receptor (ATR1) gene and essential hypertension in the south west Region of Cameroon. Int. J. Clin. Exp. Med. 2016, 9, 4071-4076.

42. Farrag, W.; Eid, M.; El-Shazly, S.; Abdallah, M. Angiotensin II type 1 receptor gene polymorphism and telomere shortening in essential hypertension. Mol. Cell. Biochem. 2011, 351, 13-18. [CrossRef]

43. Williams, S.M.; Ritchie, M.D.; Iii, J.A.P.; Dawson, E.; Prince, M.; Dzhura, E.; Willis, A.; Semenya, A.; Summar, M.; White, B.C.; et al. Multilocus Analysis of Hypertension: A Hierarchical Approach. Hum. Hered. 2004, 57, 28-38. [CrossRef]

44. Zawilla, N.; Shaker, D.; Abdelaal, A.; Aref, W. Angiotensin-converting enzyme gene polymorphisms and hypertension in occupational noise exposure in Egypt. Int. J. Occup. Environ. Health 2014, 20, 194-206. [CrossRef]

45. Abdelnour, J.G.W.; Abdelnour, Y.G.W.; Kerollos, R.-M.A.B.; Mahmoud, Z.I.T. Parry-Romberg syndrome associated with en coup de sabre in a patient from South Sudan-A rare entity from East Africa: A case report. J. Med Case Rep. 2019, 13, 138. [CrossRef]

46. Bessa, S.S.; Ali, E.M.; Hamdy, S.M. The role of glutathione S- transferase M1 and T1 gene polymorphisms and oxidative stress-related parameters in Egyptian patients with essential hypertension. Eur. J. Intern. Med. 2009, 20, 625-630. [CrossRef] 
47. Amrani-Midoun, A.; Kiando, S.R.; Treard, C.; Jeunemaitre, X.; Bouatia-Naji, N. Genetic association study between T-786C NOS3 polymorphism and essential hypertension in an Algerian population of the Oran city. Diabetes Metab. Syndr. Clin. Res. Rev. 2019, 13, 1317-1320. [CrossRef]

48. Gamil, S.; Erdmann, J.; Abdalrahman, I.B.; Mohamed, A.O. Association of NOS3 gene polymorphisms with essential hypertension in Sudanese patients: A case control study. BMC Med Genet. 2017, 18, 128. [CrossRef]

49. Jemaa, R.; Kallel, A.; Sediri, Y.; Omar, S.; Feki, M.; Elasmi, M.; Haj-Taïeb, S.; Sanhaji, H.; Kaabachi, N. Association between -786TC polymorphism in the endothelial nitric oxide synthase gene and hypertension in the Tunisian population. Exp. Mol. Pathol. 2011, 90, 210-214. [CrossRef]

50. Sediri, Y.; Kallel, A.; Ayadi, I.; Feki, M.; Elasmi, M.; Taieb, S.H.; Sanhaji, H.; Souheil, O.; Jemaa, R.; Kaabachi, N. Lack of association between endothelial nitric oxide synthase gene G894T polymorphism hypertension in the Tunisian population. Prev. Med. 2010, 51, 88-89. [CrossRef]

51. Jemaa, R.; Ben Ali, S.; Kallel, A.; Feki, M.; Elasmi, M.; Taieb, S.H.; Sanhaji, H.; Omar, S.; Kaabachi, N. Association of a 27-bp repeat polymorphism in intron 4 of endothelial constitutive nitric oxide synthase gene with hypertension in a Tunisian population. Clin. Biochem. 2009, 42, 852-856. [CrossRef]

52. Amrani-Midoun, A.; Kiando, S.R.; Treard, C.; Jeunemaitre, X.; Bouatia-Naji, N. The relationship between MTHFR C677T gene polymorphism and essential hypertension in a sample of an Algerian population of Oran city. Int. J. Cardiol. 2016, 225, 408-411. [CrossRef]

53. Lardjam-Hetraf, S.A.; Mediene-Benchekor, S.; Ouhaibi-Djellouli, H.; Meroufel, D.N.; Boulenouar, H.; Hermant, X.; Hamani-Medjaoui, I.; Saïdi-Mehtar, N.; Amouyel, P.; Houti, L.; et al. Effects of established blood pressure loci on blood pressure values and hypertension risk in an Algerian population sample. J. Hum. Hypertens. 2014, 29, 296-302. [CrossRef]

54. Nassereddine, S.; Kassogué, Y.; Korchi, F.; Habbal, R.; Nadifi, S. Association of methylenetetrahydrofolate reductase gene (C677T) with the risk of hypertension in Morocco. BMC Res. Notes 2015, 8, 1-5. [CrossRef]

55. Amin, H.A.-K.; Aziz, H.F.A.; Leheta, O.F.; Kamal, H. Methylenetetrahydrofolate reductase (677c/t) polymorphism in myocardial infarction and hypertension. Am. J. Biochem. Biotechnol. 2012, 8, 150-156. [CrossRef]

56. Sile, S.; Velez, D.R.; Gillani, N.B.; Narsia, T.; Moore, J.H.; George, A.L.; Vanoye, C.G.; Williams, S.M. CLCNKB-T481S and essential hypertension in a Ghanaian population. J. Hypertens. 2009, 27, 298-304. [CrossRef] [PubMed]

57. Sile, S.; Gillani, N.B.; Velez, D.R.; Vanoye, C.G.; Yu, C.; Byrne, L.M.; Gainer, J.V.; Brown, N.J.; Williams, S.M.; George, A.L., Jr. Functional BSND variants in essential hypertension. Am. J. Hypertens. 2007, 20, 1176-1182. [CrossRef]

58. Jones, E.S.; Owen, E.P.; Rayner, B.L. The Association of the R563Q Genotype of the ENaC With Phenotypic Variation in Southern Africa. Am. J. Hypertens. 2012, 25, 1286-1291. [CrossRef]

59. Nkeh, B.; Samani, N.J.; Badenhorst, D.; Libhaber, E.; Sareli, P.; Norton, G.R.; Woodiwiss, A.J. T594M variant of the epithelial sodium channel $\beta$-subunit gene and hypertension in individuals of African ancestry in South Africa. Am. J. Hypertens. 2003, 16, 847-852. [CrossRef]

60. Ouatou, S.; Ajjemami, M.; Charoute, H.; Sefri, H.; Ghalim, N.; Rhaissi, H.; Benrahma, H.; Barakat, A.; Rouba, H. Association of APOA5 rs662799 and rs3135506 polymorphisms with arterial hypertension in Moroccan patients. Lipids Health Dis. 2014, 13, 60. [CrossRef]

61. Lasram, K.; Ben Halim, N.; Benrahma, H.; Mediene-Benchekor, S.; Arfa, I.; Hsouna, S.; Kefi, R.; Jamoussi, H.; Ben Ammar, S.; Bahri, S.; et al. Contribution of CDKAL1 rs7756992 and IGF2BP2 rs4402960 polymorphisms in type 2 diabetes, diabetic complications, obesity risk and hypertension in the T unisian population. J. Diabetes 2014, 7, 102-113. [CrossRef] [PubMed]

62. Van Deventer, C.A.; Louw, R.; Van Der Westhuizen, F.H.; Vorster, C.B.; Malan, L. The Contribution of the C-824T Tyrosine Hydroxylase Polymorphism to the Prevalence of Hypertension in a South African Cohort: The SABPA Study. Clin. Exp. Hypertens. 2013, 35, 614-619. [CrossRef]

63. Moholisa, R.R.; Rayner, B.R.; Owen, E.P.; Schwager, S.L.U.; Stark, J.S.; Badri, M.; Cupido, C.L.; Sturrock, E.D. Association of B2 Receptor Polymorphisms and ACE Activity With ACE Inhibitor-Induced Angioedema in Black and Mixed-Race South Africans. J. Clin. Hypertens. 2013, 15, 413-419. [CrossRef]

64. Saidi, S.; Mahjoub, T.; Almawi, W.Y. Aldosterone synthase gene (CYP11B2) promoter polymorphism as a risk factor for ischaemic stroke in Tunisian Arabs. J. Renin-Angiotensin-Aldosterone Syst. 2010, 11, 180-186. [CrossRef]

65. Ben Ali, S.; Kallel, A.; Ftouhi, B.; Sediri, Y.; Feki, M.; Slimane, H.; Jemaa, R.; Kaabachi, N. The-2548G/A LEP polymorphism is associated with blood pressure in Tunisian obese patients. Blood Press 2008, 17, 278-283. [CrossRef]

66. Barlassina, C.; Norton, G.R.; Samani, N.J.; Woodwiss, A.J.; Candy, G.C.; Radevski, I.; Citterio, L.; Bianchi, G.; Cusi, D. $\alpha$-adducin polymorphism in hypertensives of South African ancestry. Am. J. Hypertens. 2000, 13, 719-723. [CrossRef] [PubMed]

67. Kabadou, I.A.; Soualmiaa, H.; Jemaa, R.; Feki, M.; Kallel, A.; Souheil, O.; Taieb, S.H.; Sanhaji, H.; Kaabachi, N. Lack of association between C3123A polymorphism of the angiotensin II type 2 receptor gene and hypertension in Tunisian population. Tunisie Medicale 2012, 90, 619-624.

68. Bis, J.C.; Sitlani, C.; Irvin, R.; Avery, C.L.; Smith, A.V.; Sun, F.; Evans, D.S.; Musani, S.K.; Li, X.; Trompet, S.; et al. Drug-Gene Interactions of Antihypertensive Medications and Risk of Incident Cardiovascular Disease: A Pharmacogenomics Study from the CHARGE Consortium. PLoS ONE 2015, 10, e0140496. [CrossRef] 
69. Agyemang, C.; Nyaaba, G.; Beune, E.; Meeks, K.; Owusu-Dabo, E.; Addo, J.; Aikins, A.D.-G.; Mockenhaupt, F.P.; Bahendeka, S.; Danquah, I.; et al. Variations in hypertension awareness, treatment, and control among Ghanaian migrants living in Amsterdam, Berlin, London, and nonmigrant Ghanaians living in rural and urban Ghana-the RODAM study. J. Hypertens. 2018, 36, 169-177. [CrossRef]

70. Oliveira-Paula, G.H.; Pereira, S.C.; Tanus-Santos, J.E.; Lacchini, R. Pharmacogenomics and Hypertension: Current Insights. Pharmacogenom. Pers. Med. 2019, 12, 341-359. [CrossRef] [PubMed]

71. El Rouby, N.; Cooper-DeHoff, R.M. Genetics of resistant hypertension: A novel pharmacogenomics phenotype. Curr. Hypertens. Rep. 2015, 17, 71. [CrossRef] [PubMed]

72. Gaddam, K.K.; Nishizaka, M.K.; Pratt-Ubunama, M.N.; Pimenta, E.; Aban, I.; Oparil, S.; Calhoun, D.A. Characterization of resistant hypertension: Association between resistant hypertension, aldosterone, and persistent intravascular volume expansion. Arch. Intern. Med. 2008, 168, 1159-1164. [CrossRef]

73. Young, A.; Ferrier, I.; Ball, S.; Mohiuddin, M.; Todhunter, C.; Mansfield, J. Genome-wide association study of 14,000 cases of seven common diseases and 3000 shared controls. Nature 2007, 447, 661.

74. Ehret, G.B.; Munroe, P.B.; Rice, K.M.; Bochud, M.; Johnson, A.D.; Chasman, D.I.; Albert, V.S.; Martin, D.T.; Germaine, C.V.; Shih-Jen, H.; et al. Genetic variants in novel pathways influence blood pressure and cardiovascular disease risk. Nature 2011, 478, 103. [PubMed]

75. Franceschini, N.; Fox, E.; Zhang, Z.; Edwards, T.L.; Nalls, M.A.; Sung, Y.J.; Tayo, B.O.; Sun, Y.V.; Gottesman, O.; Adeyemo, A.; et al. Genome-wide Association Analysis of Blood-Pressure Traits in African-Ancestry Individuals Reveals Common Associated Genes in African and Non-African Populations. Am. J. Hum. Genet. 2013, 93, 545-554. [CrossRef] [PubMed]

76. Kato, N.; Takeuchi, F.; Tabara, Y.; Kelly, T.N.; Go, M.J.; Sim, X.; Tay, W.T.; Chen, C.-H.; Zhang, Y.; Yamamoto, K.; et al. Meta-analysis of genome-wide association studies identifies common variants associated with blood pressure variation in east Asians. Nat. Genet. 2011, 43, 531-538. [CrossRef] [PubMed]

77. Kelly, T.N.; Takeuchi, F.; Tabara, Y.; Edwards, T.L.; Kim, Y.J.; Chen, P.; Li, H.; Wu, Y.; Yang, C.-F.; Zhang, Y.; et al. Genome-Wide Association Study Meta-Analysis Reveals Transethnic Replication of Mean Arterial and Pulse Pressure Loci. Hypertension 2013, 62, 853-859. [CrossRef]

78. Mengesha, H.G.; Petrucka, P.; Spence, C.; Tafesse, T.B. Effects of angiotensin converting enzyme gene polymorphism on hypertension in Africa: A meta-analysis and systematic review. PLoS ONE 2019, 14, e0211054. [CrossRef]

79. Mehri, S.; Mahjoub, S.; Hammami, S.; Zaroui, A.; Frih, A.; Betbout, F.; Mechmeche, R.; Hammami, M. Renin-angiotensin system polymorphisms in relation to hypertension status and obesity in a Tunisian population. Mol. Biol. Rep. 2011, 39, $4059-4065$. [CrossRef]

80. Shesely, E.G.; Maeda, N.; Kim, H.-S.; Desai, K.M.; Krege, J.H.; Laubach, V.E.; Sherman, P.A.; Sessa, W.C.; Smithies, O. Elevated blood pressures in mice lacking endothelial nitric oxide synthase. Proc. Natl. Acad. Sci. USA 1996, 93, 13176-13181. [CrossRef]

81. Cho, Y.S.; Go, M.J.; Kim, Y.J.; Heo, J.Y.; Oh, J.H.; Ban, H.-J.; Yoon, D.; Lee, M.H.; Kim, D.-J.; Park, M.; et al. A large-scale genome-wide association study of Asian populations uncovers genetic factors influencing eight quantitative traits. Nat. Genet. 2009, 41, 527-534. [CrossRef] [PubMed]

82. Levy, D.; Ehret, G.B.; Rice, K.; Verwoert, G.C.; Launer, L.J.; Dehghan, A.; Glazer, N.L.; Morrison, A.C.; Johnson, A.D.; Aspelund, T.; et al. Genome-wide association study of blood pressure and hypertension. Nat. Genet. 2009, 41, 677-687. [CrossRef] [PubMed]

83. Olson, S.; Wang, M.G.; Carafoli, E.; Strehler, E.E.; McBride, O. Localization of two genes encoding plasma membrane Ca2+transporting ATPases to human chromosomes 1q25-32 and 12q21-23. Genomics 1991, 9, 629-641. [CrossRef]

84. Xu, J.; Qian, H.-X.; Hu, S.-P.; Liu, L.-Y.; Zhou, M.; Feng, M.; Su, J.; Ji, L.-D. Gender-specific association of ATP2B1 variants with susceptibility to essential hypertension in the Han Chinese population. BioMed Res. Int. 2016. [CrossRef]

85. Goyette, P.; Sumner, J.S.; Milos, R.; Duncan, A.M.; Rosenblatt, D.S.; Matthews, R.G.; Rozen, R. Human methylenetetrahydrofolate reductase: Isolation of cDNA, mapping and mutation identification. Nat. Genet. 1994, 7, 195-200. [CrossRef]

86. McNulty, H.; Strain, J.; Hughes, C.F.; Ward, M. Riboflavin, MTHFR genotype and blood pressure: A personalized approach to prevention and treatment of hypertension. Mol. Asp. Med. 2017, 53, 2-9. [CrossRef]

87. Ilhan, N.; Kucuksu, M.; Kaman, D.; Ilhan, N.; Ozbay, Y. The 677 C/T MTHFR Polymorphism is Associated with Essential Hypertension, Coronary Artery Disease, and Higher Homocysteine Levels. Arch. Med Res. 2008, 39, 125-130. [CrossRef]

88. Qian, X.; Lu, Z.; Tan, M.; Liu, H.; Lu, D. A meta-analysis of association between C677T polymorphism in the methylenetetrahydrofolate reductase gene and hypertension. Eur. J. Hum. Genet. 2007, 15, 1239-1245. [CrossRef]

89. Moses, D.K.; Charles, B.G.; Ravenscroft, P.J. Proceedings of the Australasian Society of Clinical and Experimental Pharmacologists. Clin. Exp. Pharmacol. Physiol. 1983, 10, 641-730. [CrossRef]

90. El-Garawani, I.; Shaheen, E.; El-Seedi, H.; Khalifa, S.; Mersal, G.; Emara, M.; Kasemy, Z. Angiotensinogen Gene Missense Polymorphisms (rs699 and rs4762): The Association of End-Stage Renal Failure Risk with Type 2 Diabetes and Hypertension in Egyptians. Genes 2021, 12, 339. [CrossRef]

91. Edwards, I.R.; Aronson, J.K. Adverse drug reactions: Definitions, diagnosis, and management. Lancet 2000, 356, 1255-1259. [CrossRef]

92. Toyoshima, H.; Takahashi, K.; Akera, T. The impact of side effects on hypertension management: A Japanese survey. Clin. Ther. 1997, 19, 1458-1469. [CrossRef] 
93. Bardage, C.; Isacson, D.G. Self-reported side-effects of antihypertensive drugs: An epidemiological study on prevalence and impact on health-state utility. Blood Press 2000, 9, 328-334. [PubMed]

94. Grégoire, J.-P.; Moisan, J.; Guibert, R.; Ciampi, A.; Milot, A.; Gaudet, M.; Côté, I. Determinants of discontinuation of new courses of antihypertensive medications. J. Clin. Epidemiol. 2002, 55, 728-735. [CrossRef]

95. Lin, Y.-P.; Huang, Y.-H.; Yang, Y.-C.; Wu, J.-S.; Chang, C.-J.; Lu, F.-H. Adherence to antihypertensive medications among the elderly: A community-based survey in Tainan City, Southern Taiwan. Taiwan Geriatr. Gerontol. 2007, 2, 176-189.

96. Macedo, A.F.; Morgado, M.; Castelo-Branco, M.; Rolo, S.; Pereira, L. Predictors of uncontrolled hypertension and antihypertensive medication nonadherence. J. Cardiovasc. Dis. Res. 2010, 1, 196-202. [CrossRef] [PubMed]

97. Kronish, I.M.; Woodward, M.; Sergie, Z.; Ogedegbe, G.; Falzon, L.; Mann, D.M. Meta-analysis: Impact of drug class on adherence to antihypertensives. Circulation 2011, 123, 1611-1621. [CrossRef] [PubMed]

98. Brewster, L.M.; Seedat, Y.K. Why do hypertensive patients of African ancestry respond better to calciumblockers and diuretics than to ACE inhibitors and $\beta$-adrenergic blockers? Asystematic review. BMC Med. 2013, 11, 141. [CrossRef] [PubMed]

99. Boima, V.; Dele Ademola, A.; Odusola, A.O.; Agyekum, F.; Nwafor, C.E.; Cole, H.; Salako, B.L.; Ogedegbe, G.; Tayo, B.O. Factors Associated with Medication Nonadherence among Hypertensives in Ghana and Nigeria. Int. J. Hypertens. 2015. [CrossRef] [PubMed]

100. Ghazi, L.; Drawz, P. Advances in understanding the renin-angiotensin-aldosterone system (RAAS) in blood pressure control and recent pivotal trials of RAAS blockade in heart failure and diabetic nephropathy. F1000Research 2017, 6, 297. [CrossRef]

101. Yang, J.; Griffiths, M.; Nies, M.K.; Brandal, S.; Damico, R.; Vaidya, D.; Tao, X.; Simpson, C.E.; Kolb, T.M.; Mathai, S.C.; et al. Insulin-like growth factor binding protein-2: A new circulating indicator of pulmonary arterial hypertension severity and survival. BMC Med. 2020, 18, 1-11. [CrossRef] [PubMed]

102. Kumar, S.; Malik, M.A.; Goswami, S.; Sihota, R.; Kaur, J. Candidate genes involved in the susceptibility of primary open angle glaucoma. Gene 2016, 577, 119-131. [CrossRef] [PubMed]

103. Sadee, W.; Hartmann, K.; Seweryn, M.; Pietrzak, M.; Handelman, S.K.; Rempala, G.A. Missing heritability of common diseases and treatments outside the protein-coding exome. Qual. Life Res. 2014, 133, 1199-1215. [CrossRef] [PubMed]

104. Luizon, M.R.; Pereira, D.A.; Sandrim, V.C. Pharmacogenomics of Hypertension and Preeclampsia: Focus on Gene-Gene Interactions. Front. Pharmacol. 2018, 9, 168. [CrossRef] [PubMed]

105. Oh, S.S.; Galanter, J.; Thakur, N.; Pino-Yanes, M.; Barcelo, N.E.; White, M.J.; De Bruin, D.M.; Greenblatt, R.M.; Bibbins-Domingo, K.; Wu, A.H.B.; et al. Diversity in Clinical and Biomedical Research: A Promise Yet to Be Fulfilled. PLoS Med. 2015, 12, e1001918. [CrossRef]

106. Semon, M.; Nielsen, R.; Jones, M.P.; McCouch, S.R. The population structure of African cultivated rice Oryza glaberrima (Steud.): Evidence for elevated levels of linkage disequilibrium caused by admixture with O. sativa and ecological adaptation. Genetics 2005, 169, 1639-1647. [CrossRef] [PubMed]

107. Ben Ali, M.; Messaoudi, S.; Ezzine, H.; Mahjoub, T. Contribution ofeNOSVariants to the Genetic Susceptibility of Coronary Artery Disease in a Tunisian Population. Genet. Test. Mol. Biomark. 2015, 19, 203-208. [CrossRef]

108. Hooghiemstra, A.M.; Bertens, A.S.; Leeuwis, A.E.; Bron, E.E.; Bots, M.L.; Brunner-La Rocca, H.P.; de Craen, A.; van der Geest, R.J.; Greving, J.P.; Kappelle, L.J.; et al. The Missing Link in the Pathophysiology of Vascular Cognitive Impairment: Design of the Heart-Brain Study. Cerebrovasc. Dis. Extra. 2017, 7, 140-152. [CrossRef] 